\title{
Considering a dynamic impact zone for real-time railway traffic management
}

Sofie Van Thielen ${ }^{* 1}$, Francesco Corman ${ }^{2}$, and Pieter Vansteenwegen ${ }^{1}$

${ }^{1}$ KU Leuven, Leuven Mobility Research Centre - CIB, Celestijnenlaan 300, 3001 Leuven ${ }^{2}$ ETH Zurich, Institute for Transport Planning and Systems, Stefano Franscini Platz 5, Zurich 8093, Switzerland

March 21, 2018

\footnotetext{
*sofie.vanthielen@kuleuven.be
} 


\begin{abstract}
In a railway system, a conflict occurs when two trains require the same part of the infrastructure at the same time. Currently, such conflicts are typically resolved manually by experienced dispatchers. However, it is impossible for them to fully anticipate the impact of their actions on the entire network. This paper proposes a conflict prevention strategy which focuses only on the relevant part of the network and traffic and, consequently, proposes a solution for that part only. The proposed strategy first looks for possible rerouting options by using an optimization model. If no solution is found, a solution based on delaying one of the trains is required. This retiming/reordering heuristic uses information from an offline calculation, for determining related conflicts that frequently occur. In this way, a so-called dynamic impact zone is created online for each conflict. When deciding which train to delay, the potential conflicts and the incurred delays of all trains in this dynamic impact zone are taken into account.

The performance of this new Conflict Prevention Strategy is compared to a common dispatching strategy, other heuristics, and an exact method. Extensive experiments on a large part of the Belgian railway network show that by considering this dynamic impact zone the total delay can be decreased by at least $67 \%$ compared to the basic First Come, First Served decision rule. Moreover, the dynamic impact zone has a reasonable size and scales well to large networks as only the relevant conflicts and their expected consequences are considered. This makes our Dynamic Impact Zone heuristic very fast. The computation time for returning a resolution to a conflict with the proposed conflict prevention strategy is, for $95 \%$ of the conflicts, less than two seconds, and at most 26 seconds, including the creation of the dynamic impact zone of the conflict.
\end{abstract}

Keywords: Conflict prevention; Dispatching; Traffic Management System; Realtime scheduling

\title{
1 Introduction
}

In the past few decades, the interest in public transport has been growing steadily due to the increasing need for sustainable transport. Railway companies want to attract more passengers by providing a high quality train service, including a robust timetable with as few delays as possible. A timetable normally ensures that two trains do not 'interfere' (conflict) with each other, such that delays are avoided in real-time. However, during daily operations, trains can suffer delays caused by e.g. mechanical failures, a broken part of the infrastructure, or bad weather conditions. If some trains are no longer driving according to their schedule, conflicts might occur. This means that trains now require the same part of the infrastructure at the same time. In real-time, conflict detection is required to check whether a timetable is still conflict-free or contains potential conflicts in the near future. If such a conflict is detected, it has to be decided which train can use the common part of the infrastructure first or whether some trains can be rerouted to resolve the conflict. This decision involves only two trains in the vast majority of the 
cases and is typically made by the dispatchers (traffic controllers) based on their experience. Dispatchers are increasingly seeking a Decision Support System (DSS) that includes conflict detection and prevention. If a near-future conflict is detected, it can still be prevented from happening by deciding to reroute or retime one of the conflicting trains in real-time. These prevention measures are examined in a conflict prevention module. In this paper, our conflict prevention strategy, implemented in the conflict prevention module, will aim at minimizing the total secondary delay of all trains in the network. Optimizing conflict prevention strategies is still a challenging problem, both in practice and in practically oriented research. For the rerouting phase, we developed an exact optimization procedure. For the retiming/reordering phase, we created a heuristic, which we will refer to as the Dynamic Impact Zone (DIZ) heuristic.

In the literature, train movement prediction, conflict detection, and conflict prevention are sometimes solved together by using sophisticated optimization models. However, these approaches are typically limited in the size of the network that can be considered in reasonable computation times. This is due to the fundamental complexity of the problem and the fact that all trains on the network are considered, which can reach into the hundreds in a national network. In this paper, we present the DIZ heuristic which is able to focus only on those trains that are actually relevant. This focus is a necessity given that the computation time required for solving the complete optimization problem grows exponentially as the size of the network increases.

Summarizing, in this paper, we tackle the real-time railway traffic management problem. When a conflict is predicted, we want to prevent it in such a way that the total secondary delay of trains in the network is minimized. The line planning, timetable, available infrastructure, vehicles, and crews are considered fixed. The routing, order, and timing of the trains are allowed to be modified. We assume a fixed-speed model, i.e. we do not take braking and acceleration explicitly into account when facing restrictive signal aspects. For the network considered in this paper, in order to be of use in practice, the algorithm needs to be able to determine a solution in a few seconds.

The main contribution of this paper is that a very fast Conflict Prevention Strategy (CPS, in short) is presented, which is close to practice and suitable for large and complex networks. Furthermore, we show the benefits of using both the results of a set of calculations performed offline beforehand and a 'dynamic impact zone', created online for each conflict. This zone determines which other potential conflicts and trains are the most relevant to be considered by the conflict prevention strategy when determining the solution that minimizes the incurred delays on all trains. This approach delivers solutions that clearly outperform solutions generated by the First Come First Served (FCFS) decision rule and other heuristics. Moreover, since our module starts from timetable data and microscopic infrastructure data from a Traffic Management System (TMS), it can be integrated directly in such a TMS, for instance at the Belgian railway infrastructure manager Infrabel.

Section 2 starts with some important definitions required for the remainder of the paper. A literature review is included in Section 3. Section 4 describes how the real-time situation, including a TMS and a conflict prevention module, is modeled in a closed loop simulation 
and how these different components communicate. Next, the first part of our CPS, the rerouting optimization, is explained in Section 5. The second part of our CPS, the DIZ heuristic, is explained in Section 6. The performance of the CPS is extensively evaluated using a large and complex case study in Section 7. This paper is concluded in Section 8.

\section{Definitions}

A rail network can be represented by including every detail of the infrastructure, which is called microscopic. Alternatively, the network can be represented by considering no details, only stations and links, which is called macroscopic. In this paper, a microscopic model of the infrastructure is used. Rail infrastructure is composed of sequences of block sections, i.e. the part of a track between two consecutive signals. The railway infrastructure manager uses these signals as a means of communication to inform train drivers about the where-abouts of other trains. In Belgium, a signal can be either green, double yellow, or red. Green indicates that the next two block sections are free, double yellow indicates that the next block section is free but the one after that is occupied, and red indicates that the next block section is occupied. A sequence of different adjacent block sections is called a route.

In the initial timetable, trains are planned in such a way that they can always enter the next block section without slowing down. Stated otherwise, they are planned such that they always have a green signal, implying the next two block sections are free. This is a rule of thumb often applied in practice to minimize trains slowing down (at double yellow) or even stopping (at a red light) and losing time in re-accelerating.

The rail infrastructure can be divided into station areas and non-station areas. In station areas there are platforms where passengers can embark or disembark if the train has a stop in that particular station. Before and after the station platforms, there is an area in which many switches are present enabling trains to easily switch to a different platform in the station, also called a switch area (also known as interlocking area). In this paper, it is assumed that a station area includes the platforms and also the switch areas before and after these platforms.

The movement of a train through a block section is called an operation. The time required to travel through a block section is called the occupation time, and depends on the type of train, infrastructure, and rolling stock. The occupation time consists of the minimum time necessary to travel through one block section, plus a supplement which is added for safety reasons. The occupation time starts at the entrance time, i.e. the instant that the train enters the current block section and ends at the departure time, i.e. the time instant that the (head of the) train leaves the block section. The clearing time is the additional time needed for the (tail of the) train to fully leave the block section, ending at the release time. The reservation time is the safety time interval before entering a block section. A block section cannot be used by another train during the reservation time. To conclude, a train reserves a block section starting from the start of the reservation time until the release time, following the blocking time theory in Hansen and Pachl (2008). 
The timetable is assumed to be feasible or conflict-free which means that no conflicts are present in the timetable if all trains drive according to their schedule. However, external events such as (infrastructure) defects can cause delays. These kind of delays are called primary delays. Not all primary delays can be dealt with by the supplements present in the timetable, implying that not all trains can recover their entire accumulated delay(s) without affecting other trains. Inevitably, two (or more) trains will want to reserve the same part of the infrastructure at the same time, i.e. a conflict will occur. When a conflict is detected to occur in the near future, a resolution (rerouting, retiming and reordering) needs to be proactively computed and implemented. In many cases, this resolution will cause extra delays, which are called secondary delays.

Primary delayed trains can cause other trains to be delayed, which in turn can cause still other trains to be delayed, and so on. This effect is called delay propagation. The entire delay propagation following a conflict is called the impact zone of this conflict.

\section{Literature review}

Over the past years, much research has been devoted to preventing conflicts by solving the so-called conflict resolution problem $(\mathrm{CRP})$. The CRP can be solved in many different ways. Most researchers solve the CRP by finding a feasible solution without conflicts for a certain time horizon ahead by looking at possible rerouting and rescheduling options. Predicting train movements, detecting, and preventing conflicts takes place simultaneously. For an extensive review of these models, we refer to Cacchiani et al. (2014) and Corman and Meng (2013). If a microscopic model is considered, these optimization models are often based on considering alternative graphs for solving a job-shop problem with no-wait and blocking constraints in which a job represents a train and a machine represents a block section (Mascis and Pacciarelli, 2002). This model has been extended by allowing iterative rerouting using a neighborhood search (Mazzarello and Ottaviani, 2007). These developments have led to the implementation of an advanced (laboratory) DSS, called Railway traffic Optimization by Means of Alternative graphs or ROMA (D'Ariano et al., 2008). Extensions have been created by considering additional solution methods, such as tabu search (Corman et al., 2010b) and genetic algorithms (Wegele and Schnieder, 2004), different objective functions (see Corman et al., 2010b, Corman et al., 2010c, and Corman et al., 2012b), etc. Corman et al. (2012a) deal with larger networks by solving the problem for smaller parts of the network first followed by an inter-area coordination step. In this latter paper, the considered impact zone of a conflict contains a reasonable subset of the entire network, but it is static and typically larger than the dynamic impact zones considered in our paper. Another way to deal with larger networks is by approximating the network by a macroscopic model (Kecman et al., 2013). However, macroscopic approaches do not necessarily provide feasible, microscopic solutions.

Exact Mixed Integer Linear Programming (MILP) approaches have also been applied in this field (see Törnquist, 2007). Lamorgese and Mannino (2015) use a MILP approach inspired by Benders' decomposition to create a master-slave problem using a microscopic 
model within the stations and a macroscopic model for the overall network. This approach has been improved by Lamorgese et al. (2016). Currently, a heuristic decomposition approach is operational in several systems in Italy and Latvia and this approach is being validated on several other networks (see Borndörfer et al., 2017). However, it is not shown that this approach could be applied to (or could deal with) large networks.

Pellegrini et al. (2014a) introduce a different MILP approach based on a microscopic representation of the infrastructure. The resulting optimization algorithm is called RECIFEMILP (REcherche sur la Capacité des Infrastructures FErroviaires) and is able to support the dispatcher's decisions by providing both rerouting and rescheduling options. They show that rough granularity harms determining the optimal solution (based on secondary delay), thereby indicating the necessity for a microscopic approach. The solution time of the algorithms is improved in Pellegrini et al. (2014b). Because of the large number of possible rerouting alternatives, Samà et al. (2016) introduce an algorithm based on an ant colony optimization for selecting the best subset of alternative trains. The performance of RECIFE-MILP is analyzed and compared for a larger real-life network in Pellegrini et al. (2016), but is not operational in practice.

Corman et al. (2017) propose an integrated approach of combining train scheduling and delay management in real-time railway traffic control, i.e. minimizing passenger travel time by explicitly incorporating transfer connections between (delayed) trains in the optimzation process. The authors use a significant part of the Dutch rail network as a test case. Their heuristics show a relatively large optimality gap in the worst case but in reasonable computation times. In addition, these heuristics do not consider local rerouting as a possible solution.

Samà et al. (2017) use new metaheuristic approaches, capable of delivering train scheduling and routing decisions. They are tested on different busy networks, and deliver good results both with regards to solution quality and computation time. However, these approaches are not shown to be effective on larger networks.

Meng and Zhou (2011) present a MILP approach that can be applied to track blockages. In Meng and Zhou (2014) a macroscopic stochastic program is introduced in which rerouting and rescheduling are performed simultaneously. However, the network is limited to a general N-track rail network, i.e. a corridor structure with few interconnections. Moreover, solutions are on a macroscopic level and the calculations take a considerable amount of time (more than one minute).

Caimi et al. (2012) design a train rescheduling model based on a constrained combinatorial assignment problem for solving the CRP within a time horizon of 20 minutes. They use a discrete-event model to simulate the real-time situation. However, the studied network is limited to the station area of Berne.

Recently, Quaglietta et al. (2016) introduce a framework for the automatic real-time railway traffic management, called ON-TIME, including the two solvers ROMA and RECIFE. Tests are performed on different networks. Results show that large improvements can be made when solving the CRP using a conflict detection and resolution algorithm. However, results show large differences based on different objective functions. This framework is not tested yet on larger, complex networks. 
Many railway infrastructure managers have advanced software available for predicting train movements and detecting conflicts (see Dolder et al., 2009). At Infrabel, this software is operational in a Traffic Management System (TMS), accessible by dispatchers in order to support their decisions. However, a conflict prevention module is not yet included in the current TMS, leading to the research presented in this paper.

In our paper, a discrete-event model is implemented to simulate the real-life execution of the (replanned) timetable. Predictions are carried out such that conflicts can be detected in time and conflict prevention strategies can be applied to ensure that those conflicts will not take place. In the envisaged real-life implementation, the conflict detection part of the problem will be taken care of by external modules. Therefore, this paper presents a proactive Conflict Prevention Strategy (CPS) that can easily be embedded into a TMS and which provides direct decision support for dispatchers. The CPS considers microscopic infrastructure data and includes a rerouting and a retiming/reordering part, both looking for the lowest total secondary delay. Moreover, extensive experiments on a large and complex part of the Belgian rail network show that our CPS is fast and scalable, and thus suited for these kind of networks. This paper only considers small delays or disturbances, thus no complete blockages where some part of the infrastructure cannot be used for a longer time.

We assume a fixed-speed model which includes planned acceleration and deceleration as in the timetable, but does not consider further changes in the speed profiles as a consequence of conflicts (e.g., Cacchiani et al. (2014)). Referring to papers that discuss to some extent the differences between variable-speed models and fixed-speed models (D'Ariano et al., 2007; Corman et al., 2010a; Sobieraj et al., 2011; Quaglietta et al., 2016; Wang and Goverde, 2017; Luan et al., 2017), we expect that the conflict prevention strategy presented in this paper would still outperform basic dispatching strategies (though the gap between different strategies might change to some extent).

In a previous conference paper (Van Thielen et al., 2017), we presented preliminary results of an initial version of the proposed conflict prevention strategy. However, this strategy did not yet consider a dynamic impact zone nor offline calculations. The previous work focused on analyzing multiple conflicts simultaneously and the effect of changing the prediction horizon in the prediction module.

\section{Closed Loop setup}

This section describes how the conflict prevention strategy is tested. A closed loop setup is considered, where three components model a complete TMS interacting with real life (see also Corman and Quaglietta, 2015). The three components are a simulator module, a predictor module, and a conflict prevention module.

Figure 1 gives an overview of how these different modules communicate with each other. The three modules all use time steps of one second. The simulator module consists of a discrete event simulation based on microscopic infrastructure data and a real microscopic timetable with a precision of one second. This module resembles reality in the sense that 
it takes into account stochastic dynamics of delays, and progresses synchronously in time. The simulator module starts at a given start hour, for example at 7 a.m. It will evaluate the performance of the conflict prevention module during the simulation horizon, which is set to 60 minutes. At the end of the simulation, the total secondary delay of all the running trains is calculated. This is the result of many iterations of conflict detection, optimization and implementation of solution measures, in a closed loop fashion. Whenever a train wants to reserve its next block section, the prediction module examines the progress of all trains during the prediction horizon, looking for conflicts. Because of large computation times for both the train movement prediction and the conflict detection modules, the prediction horizon is limited to 5 minutes. If a conflict is detected within the prediction horizon, the conflict is sent to the conflict prevention module. When resolving a conflict in the conflict prevention module, the goal is to find a resolution for the conflict that minimizes the total secondary delay of all trains in the considered network. Changes to the current situation during the calculation should not cause issues when implementing the calculated resolution. Therefore, both the rerouting optimization and the DIZ heuristic start their calculations from the expected situation one minute after the moment of detecting the conflict. Stated otherwise, no changes to ongoing operations can be executed in this minute. This minute is called the control delay, and whatever happens within this time interval, after detecting the conflict, cannot be changed by the conflict prevention module. Actually, this one minute control delay gives the conflict prevention module one minute time to calculate (and implement) a resolution.

If the conflict takes place in a station area, the rerouting optimization tries to find a conflict-free solution first. The rerouting optimization will be explained in detail in Section 5. If the rerouting optimization finds an optimal solution and thus results in alternative routes, the prediction is adapted based on this solution, which might lead to new conflicts. The advantage of using rerouting is that no retiming/reordering is required and no extra secondary delays are imposed in order to resolve the conflict. In the case that the rerouting cannot find an optimal solution resolving the conflict, or if the conflict takes place in a non-station area, the conflict needs to be tackled by finding a retiming/reordering solution. Retiming/reordering is optimized through the DIZ heuristic leading to (further) delaying one of the conflicting trains, and/or altering the order of the trains. The solution computed in this way is then implemented by locally rerouting trains and/or changing the time and order of the trains involved in the conflict. The prediction is adapted based on this solution, which might lead to new conflicts. This process is repeated until no conflicts are found within the prediction horizon. Afterwards, the discrete-event simulation synchronizes with the outcome of the conflict prevention module and proceeds until another train wants to reserve a block section and then the procedure starts again.

In principle, it could happen that resolving one conflict leads to another conflict in the current prediction horizon. Resolving this conflict could again lead to another conflict in the same prediction horizon and so on... The cumulative time to resolve all these conflicts could, in theory, become longer than the control delay or even longer than the prediction horizon. In that case, conflicts could be resolved very fast by applying FCFS. However, in all of our experiments it never happened (or even came close to happening) that the 
cumulative calculation time became longer than the control delay. This is probably due to the very short average calculation time compared to the control delay.

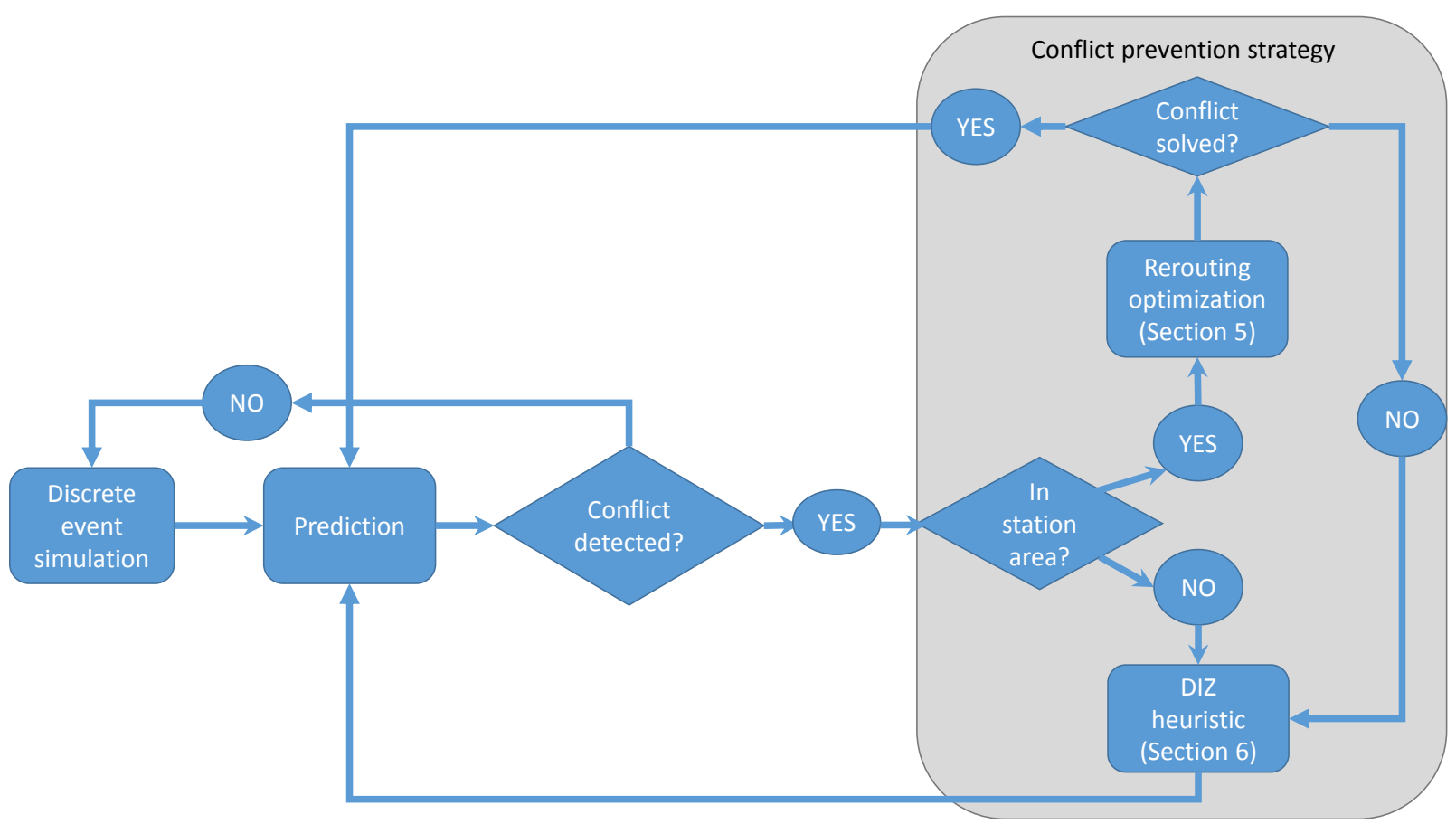

Figure 1: Flow chart of the general structure of the closed loop setup.

\section{Rerouting optimization}

In many railway networks, such as in Belgium, most rerouting options are available near and in the stations because there are typically more switches and parallel tracks allowing many alternative routes. Therefore, we limit ourselves to rerouting in station areas, i.e. local rerouting. Thus, rerouting is executed every time a detected conflict is located in a station area. The rerouting optimization procedure is based on a flexible job shop scheduling problem, which is an extension of the classical job shop scheduling problem. Trains are modelled as jobs that are operated on machines, representing the block sections (see Mascis and Pacciarelli, 2002). Trains can drive through a station area using different routes, therefore a flexible job shop problem is required. For every train, one possible route per station platform is considered in order to limit the calculation time of the optimization. In this manner, the search area is restricted and rerouting will always correspond to changing the platform in the station. This provided route per platform is the logical choice connecting its inbound and outbound route.

During the optimization, only changes inside the station area are considered, not in the rest of the network. This means that the lines on which all trains will enter and leave the station area and also the current position of the different trains already in the station 
area, are fixed. The rerouting starts from the situation in the station area, one minute after the moment of detecting the conflict (the control delay), whereas the end time is the latest departure time of one of the conflicting trains from the station area. The rerouting optimization considers all trains passing this station within this time period. As long as a train has not started its route in the station area, its planned route can be changed. If an optimal solution is found, this is implemented, preventing any conflicts to occur in the station area.

\section{Objective function}

The objective is to minimize the total secondary delay caused in the station area during the given time interval. An additional term is included into the objective ensuring the original route is preferred over an alternative route if no improvement in secondary delay is found.

\section{Parameters}

$T=\left\{t_{1}, \ldots, t_{|T|}\right\}$, set of trains with $|T|$ the number of trains.

$R=\left\{r_{1}, \ldots, r_{|R|}\right\}$, set of routes with $|R|$ the number of routes.

$R_{t} \subset R=$ set of routes train $t$ can take.

$C=\left\{\left(\left(b_{i}, t_{i}, r_{i}\right),\left(b_{j}, t_{j}, r_{j}\right)\right) \mid b_{i}\right.$ and $b_{j}$ are block sections, that share

a part of the infrastructure or that are identical, with $b_{i}$

belonging to route $r_{i}$ from train $t_{i}$ and $b_{j}$ to route $r_{j}$ from train $\left.t_{j}\right\}$

$\delta_{t}=$ earliest time instant at which train $t$ can start reserving the first block

section in the station area.

$m r_{t, b}=$ minimum reservation time required for train $t$ before entering block section $b$

$m o_{t, b}=$ minimum occupation time required for train $t$ to travel through block section $b$

$m c_{t, b}=$ minimum clearing time required for train $t$ to leave block section $b$

$n b B S_{r}=$ total amount of block sections in route $r$ of this station area.

The subset $R_{t}$ includes routes that start and end at the predetermined signals of train $t$ when, respectively, entering and leaving the station area. Note that $\delta_{t}$ is the start reservation time instant determined by the timetable and the imposed delay on train $t$ at the current time. This model also uses block section 0, indicating the block section just before the station area. 


\section{Decision variables}

$$
x_{t, r}= \begin{cases}1 & \text { if train } t \text { takes route } r \in R_{t} \\ 0 & \text { otherwise }\end{cases}
$$

$S R_{t, b}=$ actual start reservation time of train $t$ on its $b$-th block section in the station area.

$D T_{t, b}=$ actual departure time of train $t$ from its $b$-th block section in the station area.

$A R_{t, b}=$ actual release time of train $t$ on its $b$-th block section in the station area.

$S D_{t}=$ secondary delay of train $t$ measured in this station area.

Note that $S R_{t, 1}$ differs from $\delta_{t}$ in the fact that $S R_{t, 1}$ can include additional delays due to conflicts in the station area. The index 0 in variable $x_{t, 0}$ corresponds to the current route of train $t$. The non-binary decision variables are expressed in seconds.

\section{Model}

$$
\begin{aligned}
\text { minimize } & \sum_{t=1}^{|T|}\left(S D_{t}-\frac{x_{t, 0}}{100}\right) \\
\text { subject to } & S R_{t, 1} \geq \delta_{t}, \forall t \in T \\
& \text { If } x_{t, r}==1, \text { then } D T_{t, b-1} \geq S R_{t, b}+m r_{t, b}, \forall t \in T, b=1, \ldots, \mathrm{nbBS}_{r} \\
& \text { If } x_{t, r}==1 \text {, then } D T_{t, b} \geq D T_{t, b-1}+m o_{t, b}, \forall t \in T, b=1, \ldots, \mathrm{nbBS}_{r} \\
& \text { If } x_{t, r}==1, \text { then } A R_{t, b} \geq D T_{t, b}+m c_{t, b}, \forall t \in T, b=1, \ldots, \mathrm{nbBS}_{r} \\
& \text { If } x_{t, r}==1, \text { then } S D_{t}=D T_{t, \mathrm{nbBS}_{r}}-\left(\delta_{t}+\sum_{b=1}^{\text {nbBS }} m_{t, b}\right), \forall t \in T . \\
& \sum_{r \in R_{t}} x_{t, r}=1, \forall t \in T . \\
& \text { If } x_{t_{1}, r_{1}}+x_{t_{2}, r_{2}}==2, \text { then } S R_{t_{1}, b_{1}} \geq A R_{t_{2}, b_{2}} \text { or } S R_{t_{2}, b_{2}} \geq A R_{t_{1}, b_{1}}, \\
& x_{t, r} \in\{0,1\}, \forall t=1, \ldots,|T| \text { and } r \in R_{t} . \\
& \text { If } x_{t, r}==1, \text { then } S R_{t, b} \geq 0, \quad \forall t=1, \ldots,|T|, \text { and } b=1, \ldots, \mathrm{nbBS}_{r} . \\
& \text { If } x_{t, r}==1, \text { then } D T_{t, b} \geq 0, \quad \forall t=1, \ldots,|T| \text { and } b=0, \ldots, \mathrm{nbBS}_{r} . \\
& S D_{t} \geq 0, \forall t=1, \ldots,|T| .
\end{aligned}
$$

Constraints (1b) - (1e) ensure the minimal duration required for driving in the station area. Constraints (1f) state that the secondary delay of train $t$ is equal to the delay recorded in this station area. One train can have alternative routes, however, only one route per train is allowed. This is imposed by Constraints $(1 \mathrm{~g})$. Constraints $(1 \mathrm{~h})$ prevent conflicts in 
the optimal solution. In other words: if train $t_{1}$ takes route $r_{1}$ and train $t_{2}$ takes route $r_{2}$ and these routes have conflicting block sections $b_{1}$ and $b_{2}$, then train $t_{1}$ cannot enter block section $b_{1}$ before train $t_{2}$ has released block section $b_{2}$ or vice versa. These constraints are automatically linearized by Cplex using indicator constraints. Based on the preliminary experiments presented in Van Thielen et al. (2017), the computation time of the rerouting optimization is restricted to 30 seconds. If no optimal solution is found within 30 seconds, the rerouting optimization is stopped without implementing alternative routes and the DIZ heuristic is started. The rerouting optimization will never delay any of the trains.

\section{Example}

A small example is presented here to illustrate the rerouting optimization. Assume there are four trains $\left\{T_{1}, T_{2}, T_{3}, T_{4}\right\}$ departing from a certain station $\mathbf{S T}$ with four platforms (ST-I, ST-II, ST-III, ST-IV), as depicted in Figure 2. The grey blocks in Figure 2 show the block sections in the infrastructure. On the right-hand side, the original timetable is depicted with, on each line, the start of the reservation time and actual release time of the block section. The colored paths are the routes from the trains: $T_{1}$ follows the red line, $T_{2}$ the green, $T_{3}$ the blue and $T_{4}$ the purple.
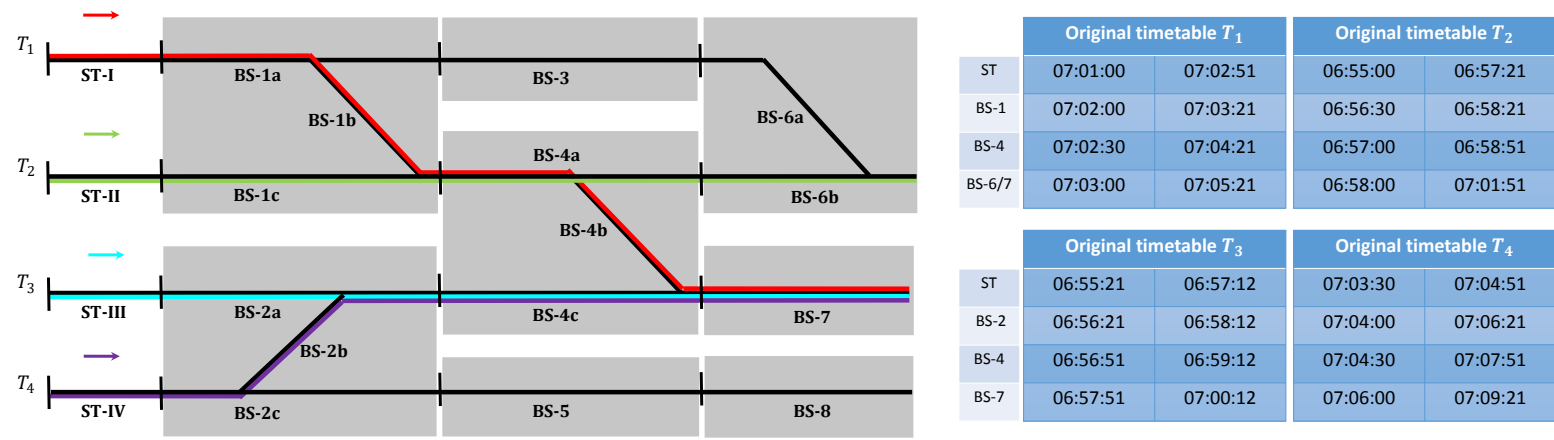

Figure 2: Example station area including four trains with the original timetable per block section.

Trains $T_{2}$ and $T_{3}$ have suffered some mechanical problems before station $\mathbf{S T}$, leading to a primary delay of respectively 5 and 8 minutes. This results in a first detected conflict between $T_{1}$ and $T_{2}$ on BS-1. Because this conflict takes place in a station area, the rerouting optimization searches for alternative routes decreasing the total secondary delay in the station area compared to no rerouting but still delaying one of these trains. All trains have to leave the station area at the same track as is the case in the original plan. For the optimization, the station area is cut after the switch area, thus only including all block sections depicted on Figure 2. Figure 3 shows the time-distance diagrams after the rerouting optimization. Trains $T_{1}$ and $T_{2}$ have switched station platforms, with routes 
BS-1c - BS-4b - BS-7 and BS-1a - BS-3 - BS-6a respectively. Trains $T_{3}$ and $T_{4}$ have kept their original route. The rerouting results in alternative routes for trains $T_{1}$ and $T_{2}$, such that they are no longer conflicting on BS-1. Trains $T_{3}$ and $T_{4}$ keep their original route. However, there still exists a conflict between trains $T_{3}$ and $T_{4}$, since all possible alternative routes only increase the total secondary delay in this station area. In this case, the rerouting solution would be discarded by our CPS, and the retiming/reordering part of the conflict prevention strategy would be triggered.

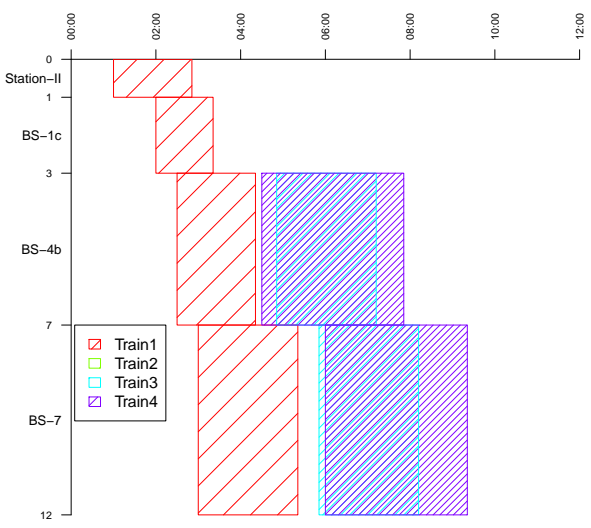

(a) $T_{1}$

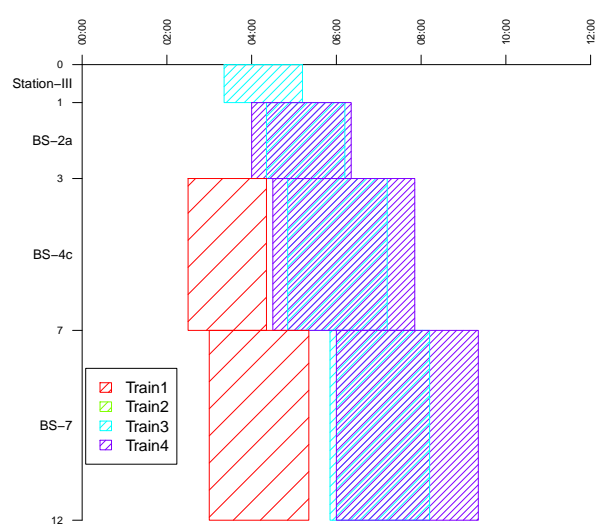

(c) $T_{3}$

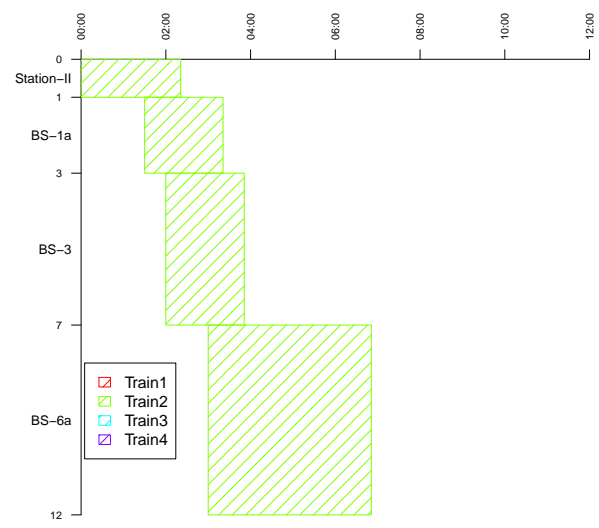

(b) $T_{2}$

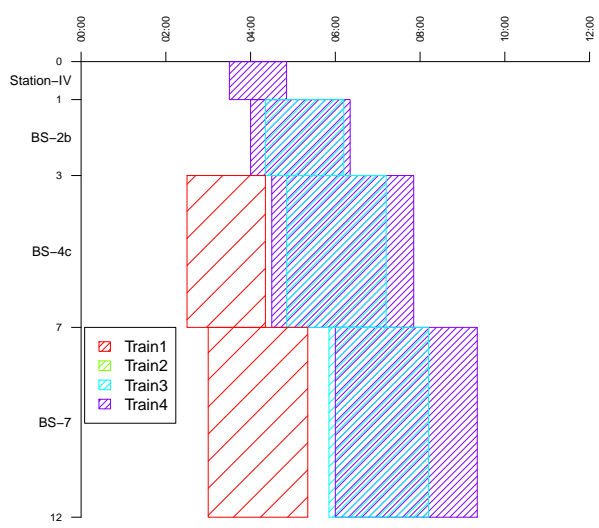

(d) $T_{4}$

Figure 3: Blocking time diagrams of trains $T_{1}, \ldots, T_{4}$ for the example, after the rerouting optimization.

\section{DIZ heuristic}

A retiming/reordering heuristic, called DIZ heuristic, is introduced in the conflict prevention module such that any (remaining) conflict can be prevented by delaying one of the conflicting trains and/or changing their order. The proposed solution should impact the 
rest of the trains as little as possible. The DIZ heuristic always results in increasing the delay of one of the conflicting trains and is based on examining the movements of the trains during the heuristic horizon, i.e. the duration over which the impact of the decision for the initial conflict is evaluated. This interval starts one minute after the moment of detecting the conflict (again considered as a control delay where no change is possible, but still well before the conflict is expected to take place), and has a fixed duration of an arbitrarily chosen 30 minutes, as shown in Figure 4. However, when the heuristic horizon ends after the simulation horizon, the end of the heuristic horizon is set to equal the end of the simulation horizon.

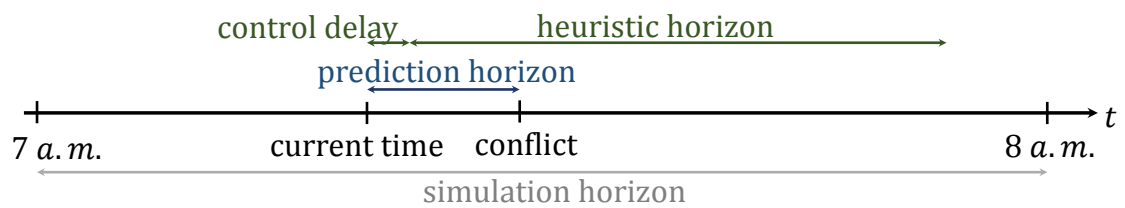

Figure 4: Different time horizons.

Before discussing in detail how the progress of the trains is examined, we first explain the difference between the initial and a new conflict and the order of the new conflicts.

\subsection{Types of conflicts}

First, it should be noted that in a conflict, the first train is the train that is predicted to arrive at the conflicting block section first. The second train is then the other train arriving at this block section.

The examination of the movements of the trains starts with the initial conflict, for which two solutions are possible: letting the first train drive first or the second. However, both cases are not necessarily possible in all situations. In fact, it could be that the two trains are already driving on the same line, where no overtaking is possible. If only one feasible option exists for the initial conflict, then the heuristic terminates immediately and returns the only possible prevention strategy.

During the examination of the further movements of both trains involved in the initial conflict, other conflicts might be detected, which are called new conflicts. These conflicts may not be detected by the prediction (yet), because these are dependent on the resolution of the initial conflict or these might take place later than the prediction horizon.

New conflicts can be classified by their degree compared to the initial conflict. Conflicts involving (at least) one of the initial conflicting trains are called first-order. Second-order conflicts are conflicts where at least one of the trains is involved in a first-order conflict, but it is not a first-order conflict itself. In this manner, an $n$ th-order conflict is a conflict of which at least one train is in an $(n-1)$ th-order conflict, but it is not an $(n-1)$ th-order conflict itself. Figure 5 shows a fictive network with an initial conflict between trains $T_{1}$ and $T_{2}$ (indicated with a square) and new conflicts discovered during the examination. The routing plans of trains $T_{i}$ are shown by putting the train's name in front and at the end of 
a line. All trains are assumed to drive from left to right. The circles represent first-order conflicts, the diamonds second-order and the triangles third-order conflicts.
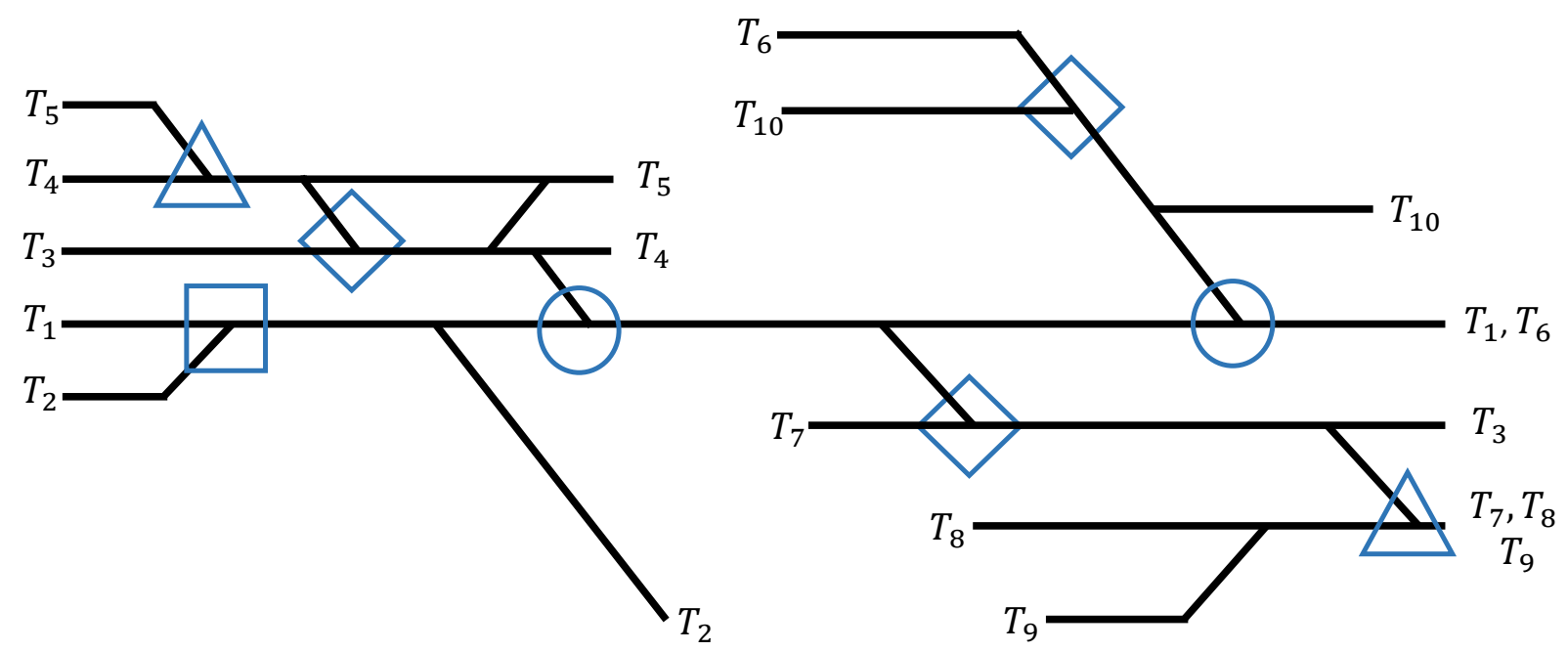

Figure 5: All possible conflicts in an example area.

\subsection{Examining the further movements of the trains}

Now we discuss in detail how the possible choices would impact the movements of the trains. This examination is required for both options: delaying the first or the second of the initial conflicting trains. The secondary delay of all trains, directly or indirectly involved in the initial conflict, will be estimated for both options. The option with the least total secondary delay is selected and returned by the heuristic. We start from a snapshot of the current operations, i.e. all trains keep the delay they have at the current time, without possibility to absorb further delays and also ignoring additional stochastic variations that might happen in the future. Obviously, based on which of the initial trains is delayed, different trains can get more or less extra delays. Ideally, the effect of (the resolutions of) all new conflicts on the progress of all the trains should be taken into account. However, determining the best resolution for all these new conflicts during the process of resolving an initial conflict would be prohibitively time consuming for realistic and large networks. Therefore, when examining the progress of the trains, some of the new conflicts will be resolved using the FCFS decision rule and other new conflicts will simply be ignored. Ignoring a conflict means that extra delay that might be caused by this new conflict is ignored, and that the delay that both trains have is assumed to remain the same after this conflict (in the case that such a potential conflict would actually occur, it would be detected at a later stage by the conflict detection module and dealt with at a later iteration). Clearly, this means the progress is not evaluated exactly for all trains, i.e. we are dealing with a heuristic. We need to make a trade-off between, on the one hand, 
resolving more of these new conflicts, thus working more accurately but also requiring more calculation time, and on the other hand, ignoring more new conflicts, requiring less computation time but maybe leading to worse decisions. This trade-off will be made by defining the dynamic impact zone.

\subsection{Dynamic impact zone}

We define the dynamic impact zone of an initial conflict as the set of all conflicts that will be considered. The conflicts in the impact zone will be resolved with FCFS when examining the consequences of both possible resolutions for the initial conflict. Therefore, the secondary delays of the trains involved in these conflicts will be determined and used to decide how to resolve the initial conflict. In preliminary research (Van Thielen et al., 2017), this impact zone was not dynamic and limited to all first-order conflicts during the remaining simulation horizon. Naturally, the impact zone can be extended by taking higher-order conflicts into account or by looking further into the future. However, this would enlarge the impact zone and it would require more computation time for the heuristic. It would be more efficient if the impact zone would only be enlarged by conflicts (and trains) that have a (large) probability of influencing the solution.

\subsubsection{Offline calculations}

To facilitate the prediction of which conflicts will significantly influence the resulting total secondary delay, carrying out offline calculations beforehand proves to be useful. These data-driven calculations are performed in order to establish which conflicts are often occurring in practice, and are thus very likely of re-occurring as soon as some trains are delayed. These calculations consist of simulation runs where random draws from delay scenarios were designed, and solved by six different strategies: FCFS, FCFS3, FCFS4, FCFS5, FCFS6 and LCFS. Preliminary results (Van Thielen et al., 2017) have shown that FCFS often does not lead to good results. Instead of always delaying the second train at a conflict, as FCFS does, further delaying the already delayed train helps to contain the delays and often turns out to be better for the entire network. This insight is used to create new rule-based strategies: FCFS3, FCFS4, FCFS5, FCFS6 and LCFS. Assume trains $T_{1}$ and $T_{2}$, respectively the first and the second train, are conflicting on a certain block section. Let delay 1 and delay $y_{2}$ stand for the accumulated delay (primary and secondary delay) of trains $T_{1}$ and $T_{2}$ up to this time instant. The starting reservation time on this block section $b$ is denoted by $S R_{1, b}$ and $S R_{2, b}$, respectively. The above strategies result then in the following rules, assuming that $S R_{1, b} \leq S R_{2, b}$ :

FCFS: delay $T_{2}$.

FCFS3: if delay $1 \geq 180 \mathrm{~s} \&$ delay $_{2}<30 \mathrm{~s}$, then delay $T_{1}$. Otherwise, delay $T_{2}$.

FCFS4: if delay $1 \geq 240 \mathrm{~s} \&$ delay $_{2}<30 \mathrm{~s}$, then delay $T_{1}$. Otherwise, delay $T_{2}$.

FCFS5: if delay $1 \geq 300 \mathrm{~s} \&$ delay $_{2}<30 \mathrm{~s}$, then delay $T_{1}$. Otherwise, delay $T_{2}$. 
FCFS6: if delay $_{1} \geq 360 \mathrm{~s} \&$ delay $_{2}<30 \mathrm{~s}$, then delay $T_{1}$. Otherwise, delay $T_{2}$.

LCFS: delay $T_{1}$.

Intuitively, the adjustments of the FCFS rule state that if one of the two trains is already delayed strongly and the other one is still on time, then it is beneficial to delay the delayed train further and let the other train continue to drive on time. The first extended rule, FCFS3, uses a time limit of 3 minutes, because this is often the minimum headway time required between two trains on the timetable level (see Sels et al., 2016). 350 random draws are taken from a given delay scenario on which these six strategies are executed. For every run (or draw) and every strategy a list of all conflicts is created. Subsequently, these lists are scanned for the most likely conflicts. The likelihood of every conflict is calculated.

For the fictive network from Section 6, Figure 6 shows the likelihood of every conflict. The most likely conflicts are conflicts occurring most of the times (over $50 \%$ ), over all strategies and delay scenarios. Only these most likely conflicts will be used when determining the impact zone. The trains involved in these conflicts are regarded as sensitive trains because they have a large probability of being in at least one conflict and thereby propagating delays over the network.

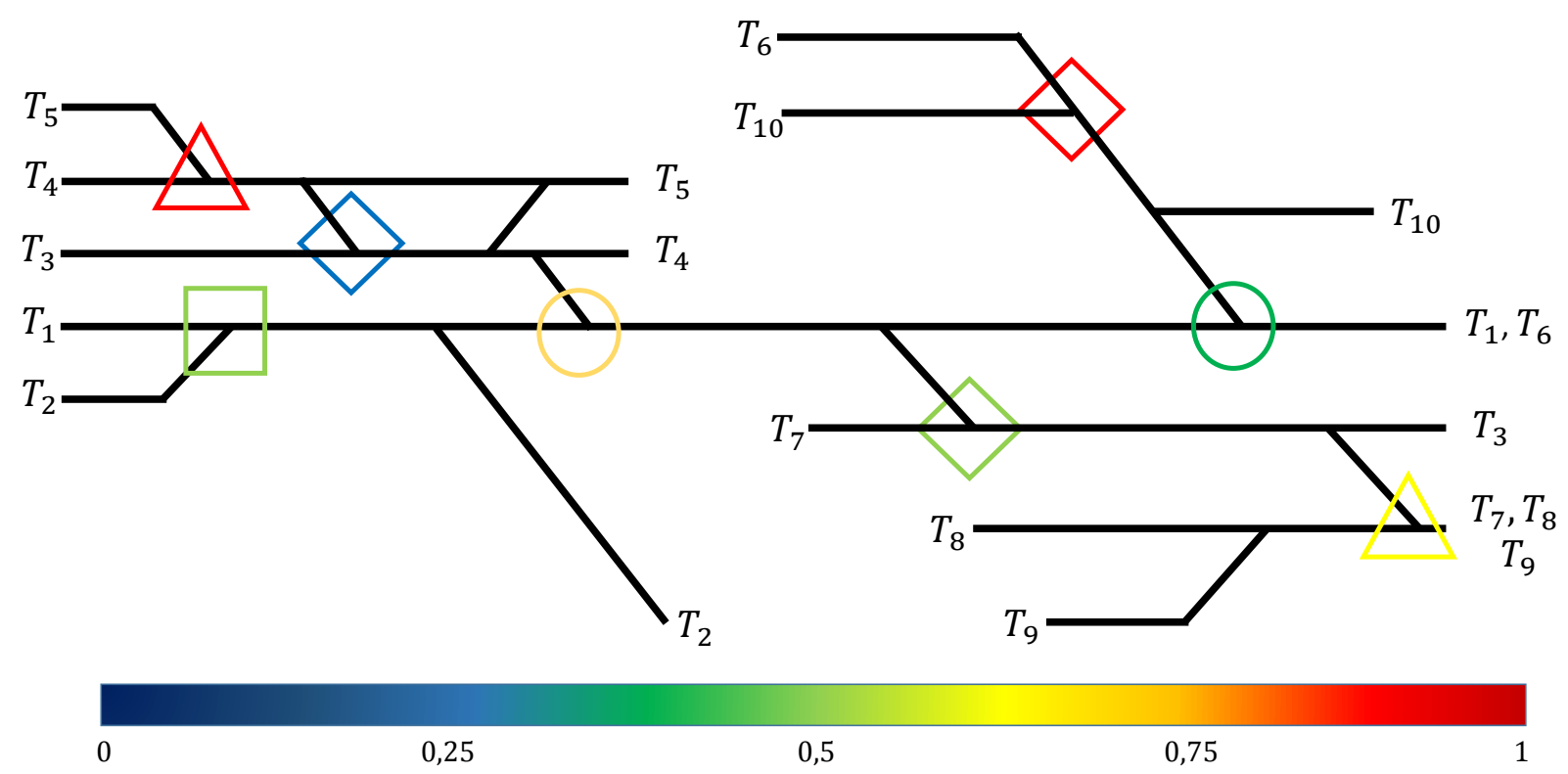

Figure 6: Likelihood of all possible conflicts in an example area.

Of course not all these most likely conflicts should always be considered when dealing with an initial conflict. The trains sharing common infrastructure within a time frame of fifteen minutes before or after a given train, are stored as potentially conflicting with this train. This calculation is also performed offline beforehand. So, for each train, a set of potentially conflicting trains is determined. In real-time, the dynamic impact zone is then 
constructed with all most likely conflicts that involve at least one of the trains in the initial conflict or a train from the sets of potentially conflicting trains of the trains of the initial conflict.

\subsubsection{Online creation of dynamic impact zone}

The DIZ heuristic always starts by determining the dynamic impact zone for the initial conflict. Both trains involved in the initial conflict are called the conflicting trains. This impact zone includes all first-order conflicts, so also the not-most likely first-order conflicts. Then, all second-order most likely conflicts, determined offline as explained above, are also added to the dynamic impact zone. When looking back at the example from Section 2, Figure 7 shows in red which conflicts will be considered during the examination of the heuristic. In this Figure, a distinction is now made between most likely conflicts (full lines) and other conflicts (dotted lines). The triangles are not considered since they are thirdorder conflicts and the blue diamond is not a most likely second-order conflict. During the examination of further movements of trains, all trains involved in these conflicts will be looked at. For this example, trains $T_{1}, T_{2}, T_{3}, T_{6}, T_{7}$ and $T_{10}$ are examined.
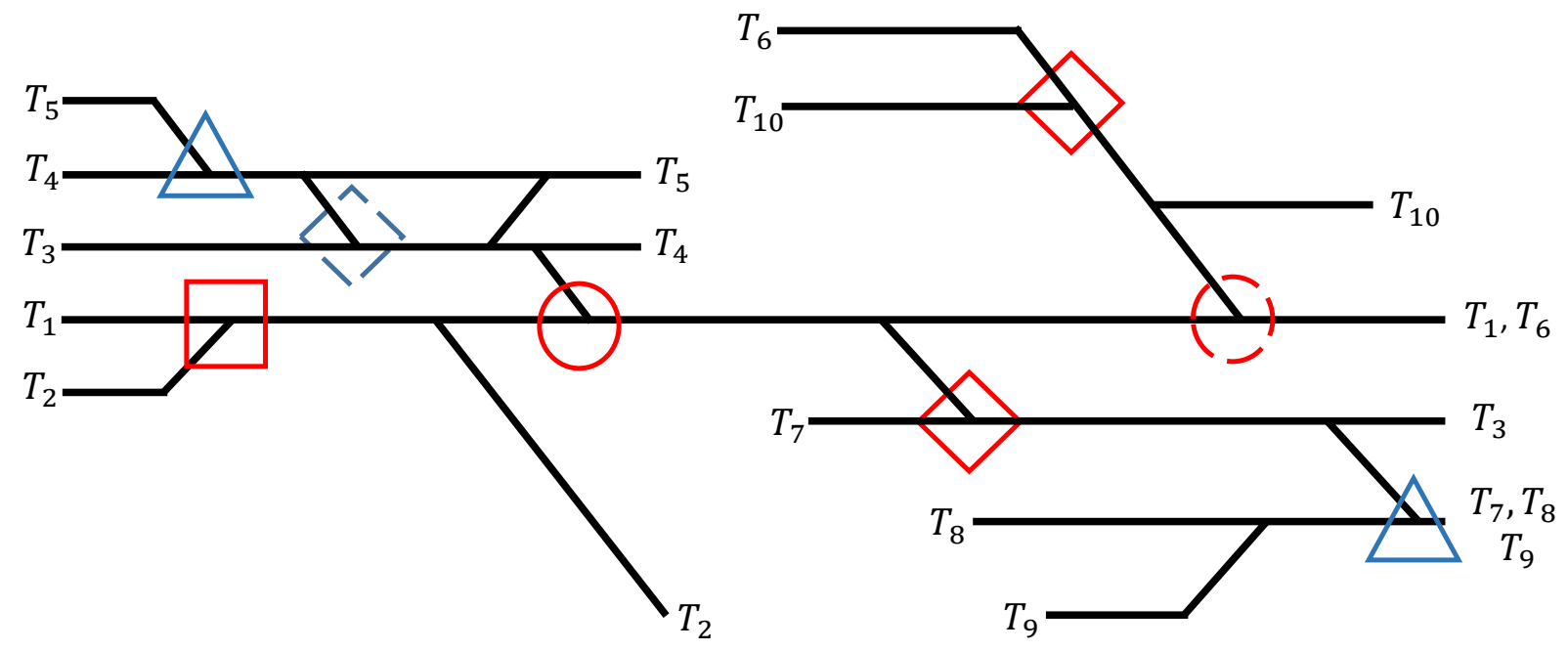

Figure 7: The most likely conflicts (full lines) and other conflicts (dotted lines) considered during the DIZ heuristic are indicated in red.

\section{Example: revisited}

Return to the simple example from the rerouting section (see Figure 2). Here, the DIZ heuristic is applied to resolve the conflict between trains $T_{3}$ and $T_{4}$. Figures $8 \mathrm{a}$ and $8 \mathrm{~b}$ 
show the resulting time distance diagram of $T_{4}$ before and after the DIZ heuristic. The full blocks indicate the delay caused by conflicts with another train. Thus, in case of the DIZ heuristic, $T_{4}$ receives a delay at the station platform in order to resolve the conflict with $T_{3}$.

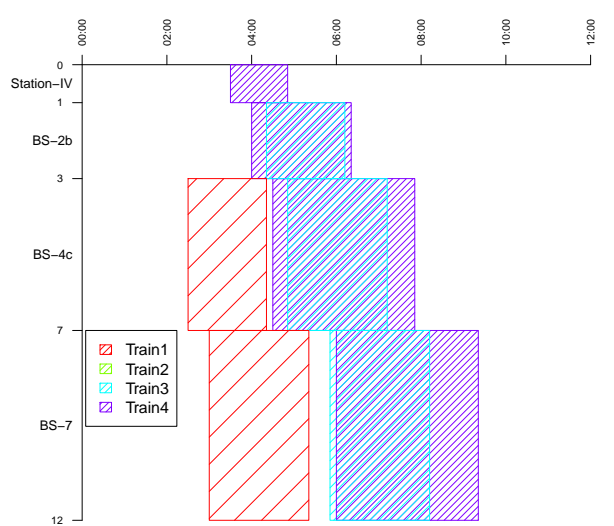

(a) Original $-T_{4}$

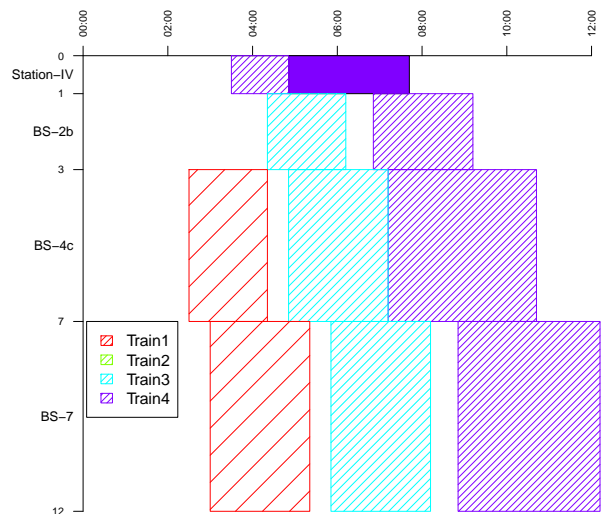

(b) DIZ - $T_{4}$

Figure 8: Blocking time diagrams for the example of $T_{4}$ before and after applying the DIZ heuristic.

\subsection{Alternative retiming/reordering heuristics}

Unfortunately, no historical data about conflict prevention in practice is available for this network. Therefore, four benchmark conflict prevention strategies are implemented, which will be used to evaluate the performance of our conflict prevention strategy.

\subsubsection{First Come, First Served}

As a first benchmark, FCFS is implemented. In a conflict, the first train wanting to reserve a block section gets priority and the second train is delayed. This is the most basic conflict prevention strategy that could be used, comparable to an unexperienced dispatcher. Obviously, our conflict prevention strategy should significantly outperform FCFS.

\subsubsection{First-order}

Secondly, the first-order strategy is a simplification of the retiming/reordering part of the conflict prevention strategy in the sense that only first-order new conflicts are included. No offline calculations are considered in this case. Still, this strategy examines the progress of both conflicting trains during the heuristic horizon. Figure 9 shows in red all the first-order conflicts in the example area. These are the conflicts that are resolved, using FCFS, by the first-order strategy during the examination of movement of trains. For the example area, 
the secondary delays of the trains $T_{1}, T_{2}, T_{3}$ and $T_{6}$ are considered when deciding how to resolve the conflict.
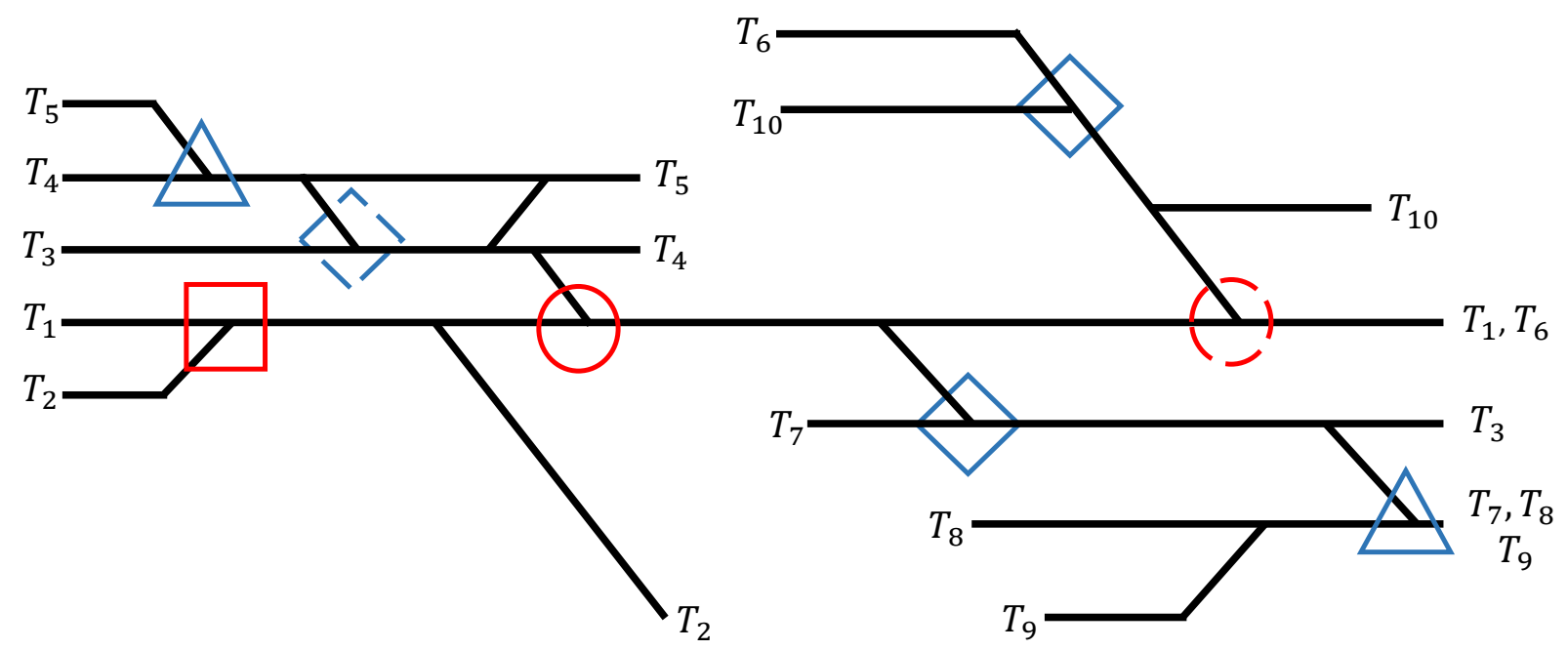

Figure 9: All first-order conflicts in the example area are indicated in red.

\subsubsection{Second-order}

The third benchmark strategy is an extension of the first-order strategy, because it considers all first-order and second-order conflicts. Therefore, this strategy includes more potential conflicts than the DIZ heuristic because the DIZ heuristic only regards first-order conflicts and most likely second-order conflicts. Figure 10 indicates in red which new conflicts are regarded for the example area. Since (much) more conflicts are considered and resolved during the heuristic, it is expected that better results are obtained compared to the firstorder strategy, but also more computation time will be required. For this example, the secondary delays of the trains $T_{1}, T_{2}, T_{3}, T_{4}, T_{6}, T_{7}$ and $T_{10}$ are considered when deciding how to resolve the conflict.

\subsubsection{Third-order}

The fourth benchmark strategy extends the second-order strategy, considering first-, secondand third-order conflicts. This strategy includes even more conflicts than the second-order strategy. Figure 11 illustrates in red which conflicts are regarded for the example area in case of the third-order strategy. All trains $T_{1}, \ldots, T_{10}$ are now considered when looking at the progress examination of the initial conflict.

The first-order, second-order and third-order strategies can be used to illustrate the benefits (or drawbacks) of working with a dynamic impact zone and offline calculations. 


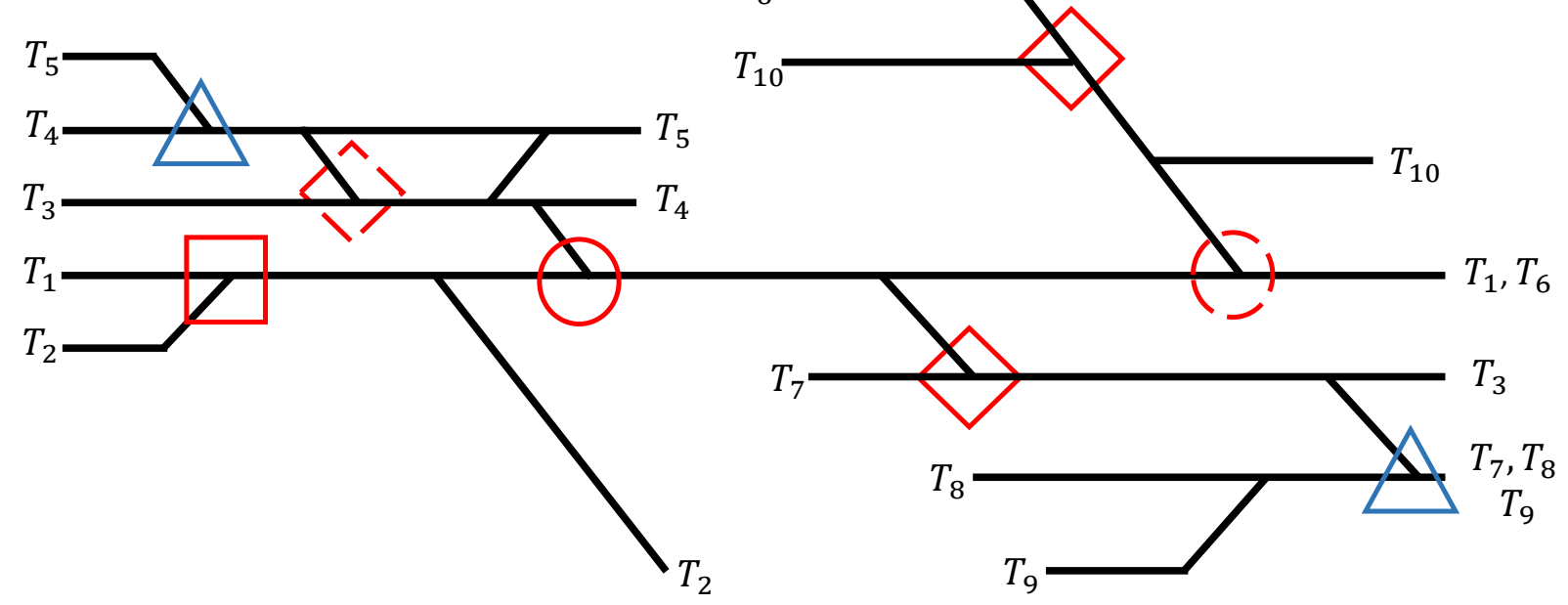

Figure 10: All first- and second-order conflicts in the example area, indicated in red.

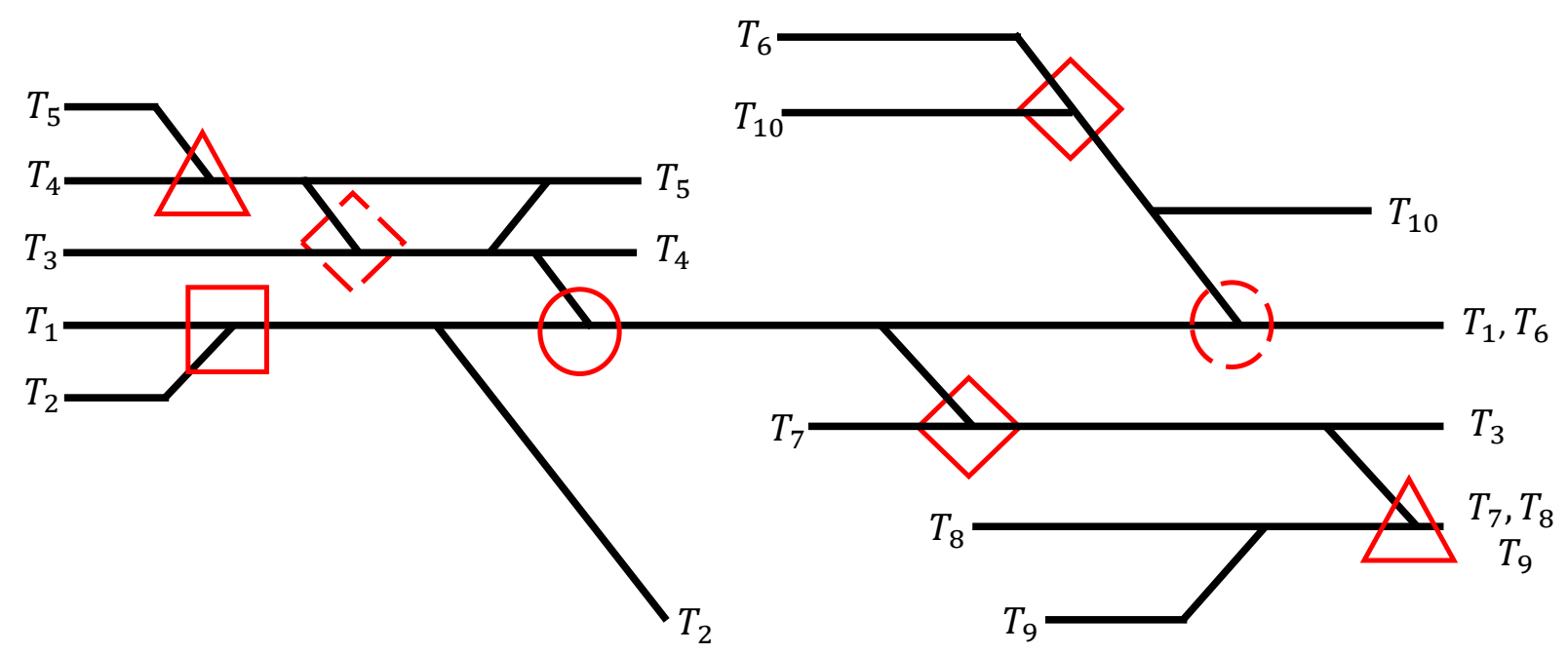

Figure 11: All first-, second- and third-order conflicts in the example area, indicated in red.

\section{$7 \quad$ Experimental results}

In order to evaluate the performance of the CPS, and for comparison with common benchmark strategies, experiments have been carried out on a large and complex network. First, 
the set-up of the case study is described. Then, the results from the offline calculations that will be used online, are reported. Subsequently, some reference case results are shown. This is followed by a sensitivity analysis. Finally, the performance of the DIZ heuristic is compared to an exact method. These tests are all carried out using an Intel(R) Core(TM) i7-3770 CPU 3.40GHz machine.

\subsection{Case study}

The performance of the new CPS is tested on the study area Brugge-Gent-Denderleeuw in Belgium (see Figure 12). This rail network is approximately $91 \mathrm{~km}$ long (OostendeDenderleeuw) and $32 \mathrm{~km}$ wide (Waregem-Gent). It consists of 84 stations having available 232 platforms. The area includes 8850 block sections. The largest stations in this area are Gent-Sint-Pieters, Oostende and Brugge, each having ten platforms. Note that the study area also includes shunting yards. The network is considered with a microscopic detail, considering switches, (automatic and manual) signals, block sections, etc. The simulation covers both passenger and freight trains. In the case study, there are 150 trains driving on this network between $7 \mathrm{a} . \mathrm{m}$. and $8 \mathrm{a} . \mathrm{m}$. Due to the type of data required and confidentiality issues, we could not apply the CPS to other complex case studies e.g. Pellegrini et al. (2014a).

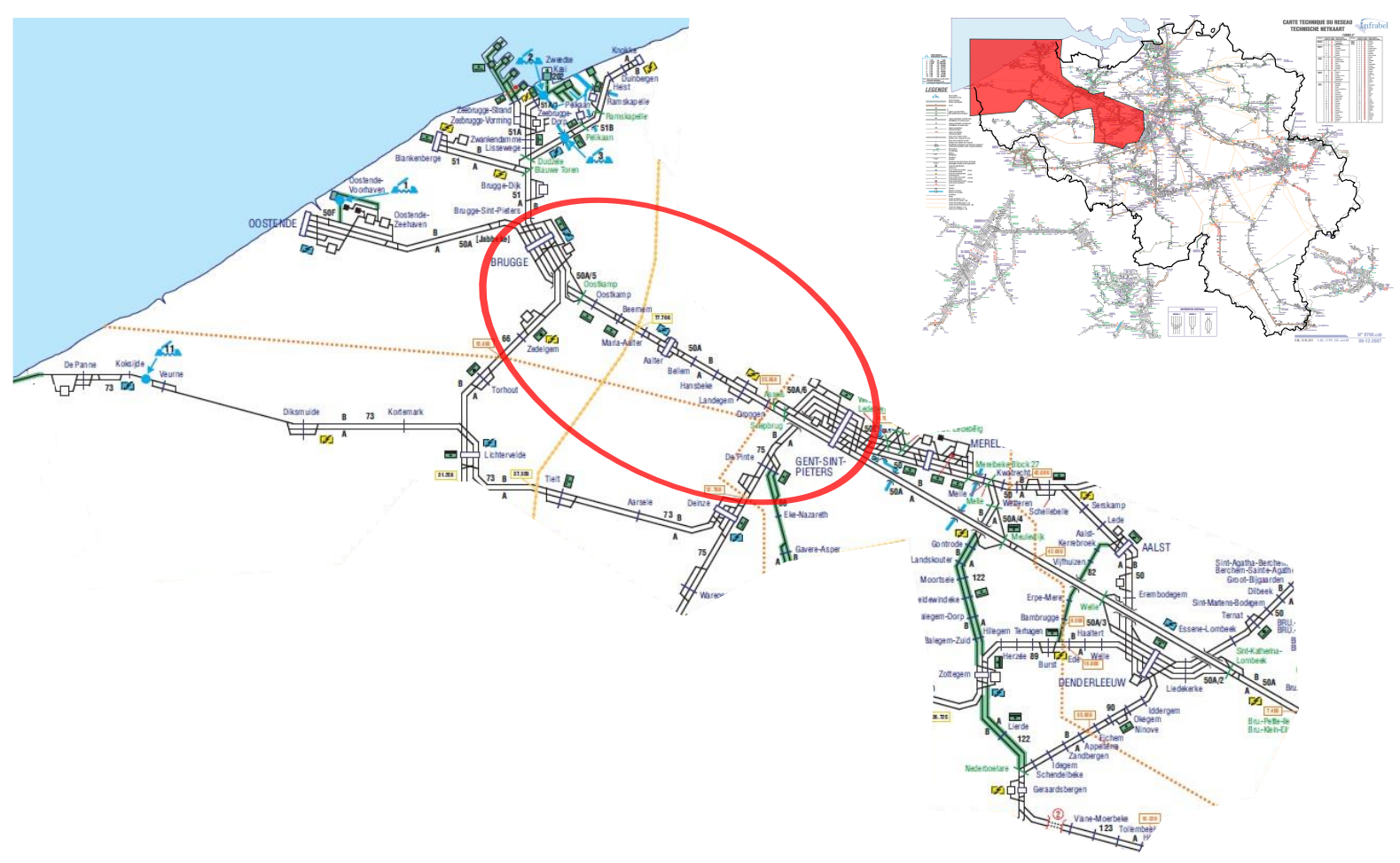

Figure 12: Study area Brugge-Gent-Denderleeuw in Belgium. The red ellipse indicates the center part of the study area between Gent and Brugge, used in Section 7.5. 
As a standard for all conflict prevention strategies, the simulation horizon is 60 minutes, the heuristic horizon is set to 30 minutes and the prediction horizon to 5 minutes. The different conflict prevention strategies are compared based on the total secondary delay, which is the secondary delay of all trains in the network, measured at the end of the simulation horizon, and the required computation time of the conflict prevention strategies.

In order to simulate conflicts in our case study, the input timetable is disturbed by drawing delays from an exponential distribution. Thus, (some of the) trains are assumed to suffer from a delay when entering the study area. A delay scenario is characterized by the percentage of delayed trains $\alpha$ (given as input), the average delay, which determines the exponential distribution, and a maximum delay, since long-time blockages or large disturbances are not considered. One run of the delay scenario takes randomly $\alpha \%$ of the trains and delays each of these trains with a number drawn from the exponential distribution with an average of 3 minutes and a maximum of 15 minutes. Ideally, instead of working with these $\alpha$ 's, historic delay scenarios could be used, but these were not available. Different disturbance levels are considered: as default we use $\alpha=60 \%$, but also values of $\alpha=20 \%, \alpha=40 \%$, and $\alpha=80 \%$ will be used. For each delay scenario (value of $\alpha$ ), 20 runs are conducted and statistically evaluated together.

\subsection{Offline calculations}

Offline calculations resulted in 83 most likely conflicts (given the cutoff threshold of $50 \%$ in this case). The likelihood of these locations is depicted in Figure 13 where every number represents the number of different most likely conflicts in the surrounded area. Clearly, some parts in the study area do not have any most likely conflict. These parts do not have a complicated infrastructure and do not use the limit of their capacity. 


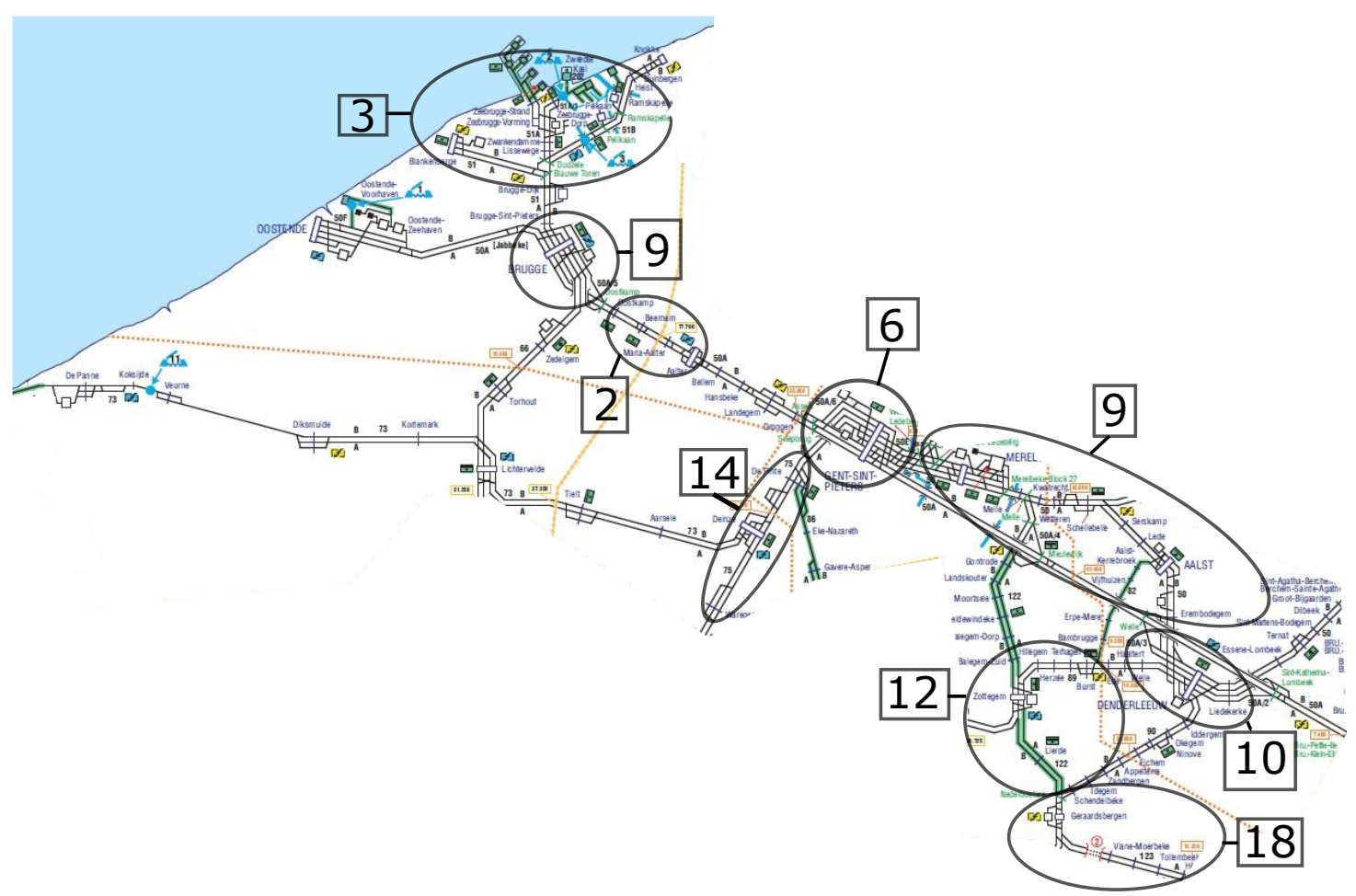

Figure 13: Most likely conflicts depicted on the study area.

\subsection{Reference case results}

The standard settings as described in Section 7.1 and the offline calculations from Section 7.2 are now used to present the reference case results. Table 1 shows the average total secondary delay over the 20 random draws from the delay scenario $\alpha=60 \%$ and the improvement compared to FCFS. Note that for every strategy, rerouting optimization is included, except for FCFS. The first-order strategy already improves the total secondary delay, but clearly the proposed conflict prevention strategy, the second-order, and the third-order strategy perform significantly better. Three one-sided paired-samples t-tests are conducted to confirm that our CPS, the second-order, and the third-order strategy indeed perform better than the first-order strategy. The three tests indicate that the null hypothesis of equality can be rejected on the significance level of $\alpha=0.05$ (after testing the assumptions necessary for the t-test, i.e. domain of the random variables, existence of categorical groups, absence of outliers, normal distribution of differences). It can be concluded that by making offline calculations, it is possible to attain similar improvements as the second-order and the third-order strategy while considering a much smaller impact zone. 


\begin{tabular}{c|c}
\hline Strategy & Total secondary delay \\
\hline FCFS & $774 \min$ \\
\hline First-order + rerouting & $436 \min (-44 \%)$ \\
Second-order + rerouting & $259 \min (-67 \%)$ \\
Third-order + rerouting & $240 \min (-69 \%)$ \\
\hline DIZ + rerouting & $252 \min (-67 \%)$ \\
\hline
\end{tabular}

Table 1: Comparison of conflict prevention techniques based on secondary delay for $\alpha=$ $60 \%$.

Table 2 shows the average computation time of preventing one conflict with the different conflict prevention strategies. This time includes the time required for the construction of the rerouting optimization and the dynamic impact zone for the DIZ heuristic, and the solution time of both. Clearly, the first-order and DIZ heuristic are very fast on average, whereas the second-order strategy requires two and a half times more computation time. The third-order strategy even requires six times more computation time. Note that the computation time of our conflict prevention strategy is, in $95 \%$ of the cases, smaller than 2 seconds. Obviously, the size of the impact zone, expressed in average number of conflicts considered (third column of Table 2), influences the computation time for determining a resolution for a detected conflict. The last column shows the percentage of those conflicts that actually need to be resolved by FCFS, when considering the further movements of the trains (i.e. the evolution of the network). The remainder of conflicts in the impact zone is also considered, but based on the train locations and timings on that moment, these conflicts will actually not have any influence on the current conflict. It should be noted that most of the computation time is spent in considering and selecting the relevant conflicts; resolving them with FCFS does not take much additional computation time. This means the computation time can be reduced by an even better selection of conflicts, only those that actually are associated to the movements of the trains under consideration. Both the first-order strategy and the DIZ heuristic actually solve approximately $10 \%$ of all conflicts in the impact zone. Clearly, the second- and third-order strategies have many conflicts in the impact zone that are not used during the evaluation of the movements of trains. Due to the long computation times and the small improvement in quality of the third-order strategy, this strategy will not be studied in the sensitivity analysis. 


\begin{tabular}{c|cccc}
\hline Strategy & $\begin{array}{c}\text { Average } \\
\text { computation } \\
\text { time }\end{array}$ & $\begin{array}{c}\text { Maximum } \\
\text { computation } \\
\text { time }\end{array}$ & $\begin{array}{c}\text { Average size impact zone } \\
\text { (numbers of conflicts } \\
\text { considered) }\end{array}$ & $\begin{array}{c}\text { Average percentage } \\
\text { conflicts resolved } \\
\text { bCFS in impact zone }\end{array}$ \\
\hline FCFS & $0.02 \mathrm{~s}$ & $0.2 \mathrm{~s}$ & - & - \\
\hline First-order + rerouting & $0.9 \mathrm{~s}$ & $25.5 \mathrm{~s}$ & 162 & $10.1 \%$ \\
Second-order + rerouting & $2.5 \mathrm{~s}$ & $29.8 \mathrm{~s}$ & 886 & $3.7 \%$ \\
Third-order + rerouting & $6 \mathrm{~s}$ & $57 \mathrm{~s}$ & 2321 & $2.2 \%$ \\
\hline DIZ + rerouting & $1 \mathrm{~s}$ & $25.8 \mathrm{~s}$ & 178 & $9.8 \%$ \\
\hline
\end{tabular}

Table 2: The computation time of the different strategies for $\alpha=60 \%$.

Figure 14 shows the locations of online detected initial conflicts for one draw of the delay scenario $\alpha=60 \%$. All strategies have approximately 160 detected initial conflicts for this draw. Figure 14 shows the initial conflicts detected when applying the DIZ heuristic. Every number in Figure 14 represents the total number of initial conflicts detected (and prevented) in the surrounded area. The locations are similar for the other strategies. Clearly, most conflicts are detected online in the same areas as where the most likely conflicts were determined offline. Obviously, some rare conflicts are also detected in different locations, depending on the randomness in the delay scenario.

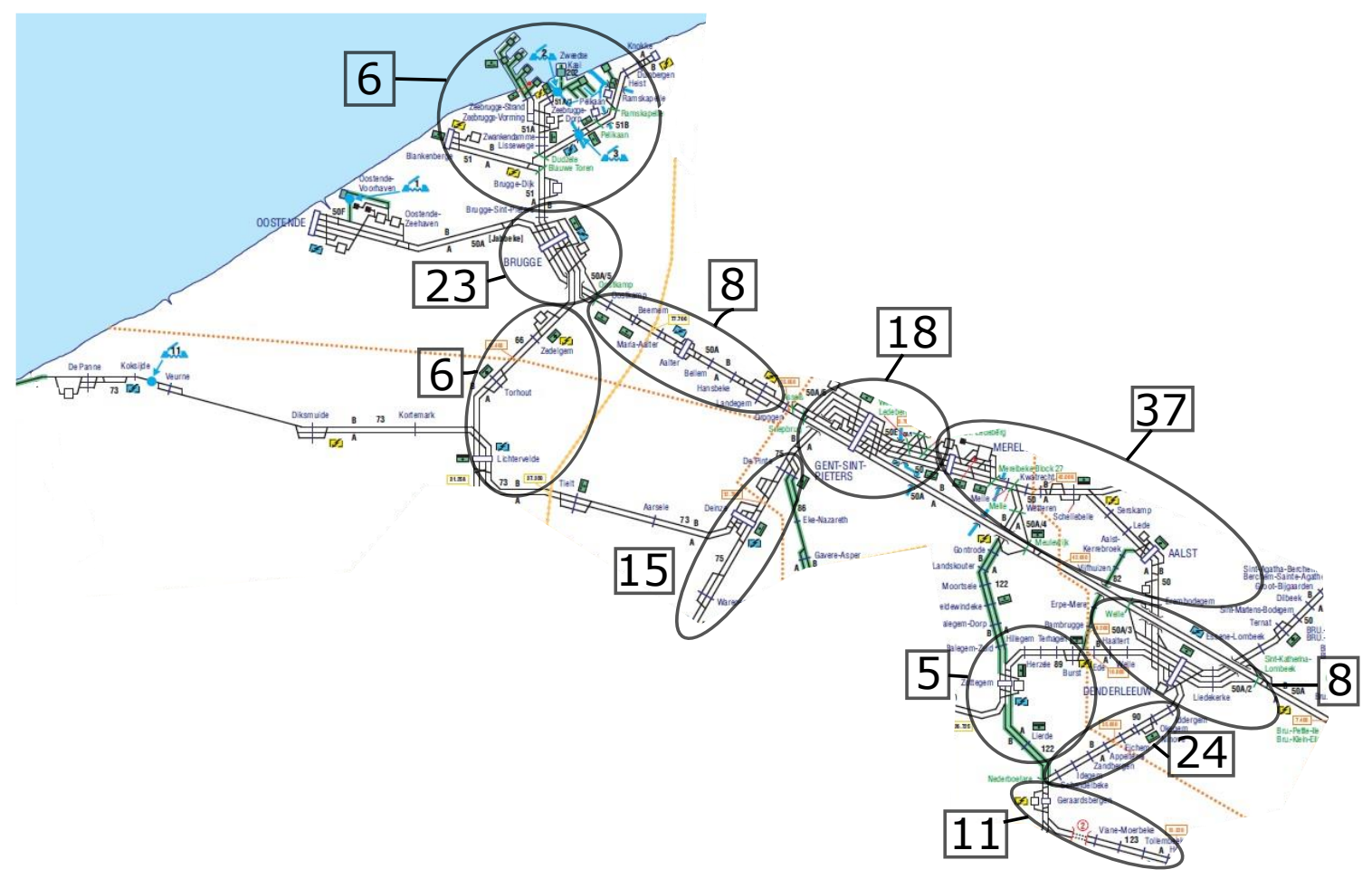

Figure 14: Locations of detected, online conflicts in case of the DIZ heuristic.

Figure 15 shows the different impact zones created for one initial conflict between 
trains 7953 and 1577, originated at the station of Geraardsbergen (indicated with a red rectangle). Every number represents the number of conflicts in the impact zone considered by the given strategy, in the surrounded area. The impact zone of the first-order heuristic includes 88 conflicts, of the second-order heuristic 1129 conflicts, of the third-order heuristic 2162 conflicts and of the DIZ heuristic 122 conflicts. Clearly, the first-order heuristic follows the train paths of both trains. Train 1577 had a final destination at the station of Geraardsbergen, whereas train 7953 drove further to Gent-Sint-Pieters. The DIZ heuristic also considers some most likely second-order conflicts. The conflicts are spread out further over the entire study area in the second-order and the third-order strategies.

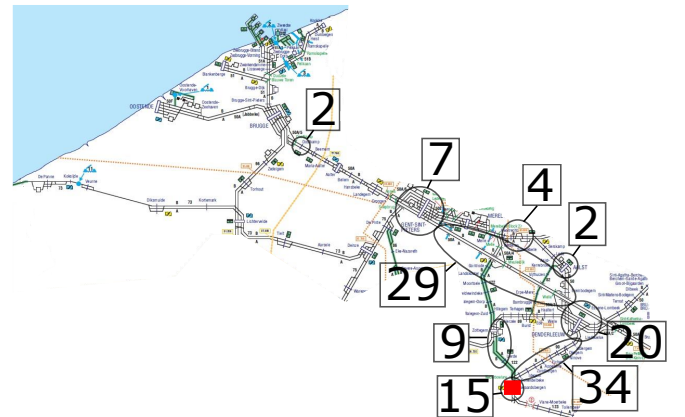

(a) DIZ heuristic

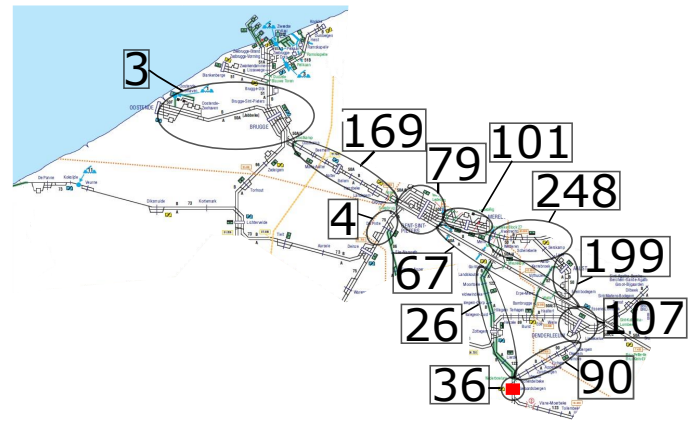

(c) Second-order

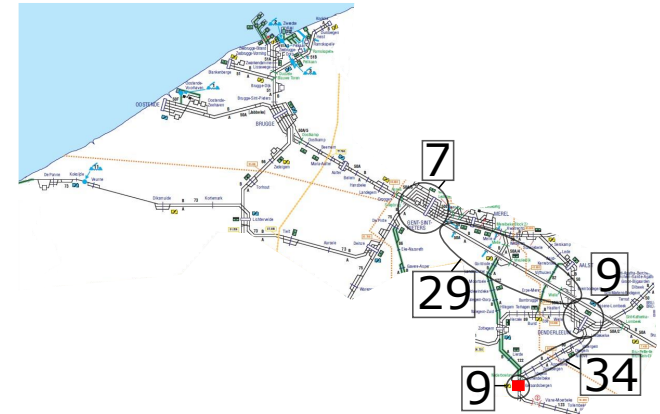

(b) First-order

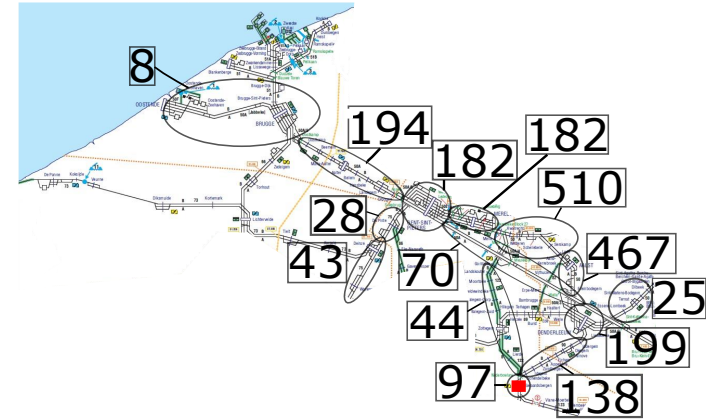

(d) Third-order

Figure 15: Example of locations of conflicts, considered in the impact zone, when resolving one initial conflict.

\subsection{Sensitivity analysis}

In this section, we analyze the performance of our strategy by changing some key characteristics, such as the heuristic horizon, leaving the rerouting optimization out, and changing the disturbance level in the delay scenario. In Van Thielen et al. (2017) we have already performed a sensitivity analysis on the prediction horizon and we concluded that changing the prediction horizon has no influence on the results. 


\subsubsection{Change in heuristic horizon}

Table 3 shows the results for a heuristic horizon (hh) of 30 minutes compared to a heuristic horizon of 60 minutes, using the same delay draws in both cases. The total secondary delay slightly deteriorates in case of the DIZ heuristic and the first- and second-order strategy. Three two-sided paired-samples t-tests are performed to confirm that there is no significant difference between the results from the heuristic horizon of 30 minutes compared to a heuristic horizon of 60 minutes. The null hypothesis cannot be rejected, in all three cases, on a significance level of $\alpha=0.05$ (after testing the assumptions necessary for the t-test). The average computation time of the strategy increases further for the secondorder strategy, up to 2.6 seconds. The other strategies render similar results in terms of computation time. Since the results are very similar, we only used a heuristic horizon of 30 minutes in all other experiments in this paper.

\begin{tabular}{c|ccc}
\hline Strategy & \multicolumn{2}{|c}{ Total secondary delay } & $\begin{array}{c}\text { Effect of longer } \\
\text { hh }=30 \mathrm{~min}\end{array}$ \\
$\mathrm{hh}=60 \mathrm{~min}$ & heuristic horizon \\
\hline FCFS & $774 \mathrm{~min}$ & $774 \mathrm{~min}$ & - \\
\hline First-order + rerouting & $436 \mathrm{~min}$ & $455 \mathrm{~min}$ & $+4.4 \%$ \\
Second-order + rerouting & $259 \mathrm{~min}$ & $283 \mathrm{~min}$ & $+9.3 \%$ \\
\hline DIZ + rerouting & $252 \mathrm{~min}$ & $277 \mathrm{~min}$ & $+9.9 \%$ \\
\hline
\end{tabular}

Table 3: Comparison of the heuristic horizon (hh) in conflict prevention techniques based on secondary delay (in $\min$ ) with $\alpha=60 \%$.

\subsubsection{Contribution of rerouting and retiming/reordering}

The same random draws from the highly disturbed case with $\alpha=60 \%$ are now run for the same strategies but omitting the rerouting part. This allows us to evaluate the impact of the rerouting part and the retiming/reordering part separately on the quality of the results and the computation time.

Clearly, the rerouting part has a significant contribution in decreasing the total secondary delays for all strategies, including FCFS (see last column in Table 4). Next to each total secondary delay, also the improvement compared to FCFS (with or without rerouting) is indicated with a percentage between brackets. It is clear that, with or without rerouting, the retiming/reordering part also contributes significantly in reducing the total secondary delay. Also here, the DIZ heuristic performs similar as the second-order strategy and both outperform the first-order strategy. 


\begin{tabular}{c|ccc}
\hline \multirow{2}{*}{ Strategy } & \multicolumn{2}{|c}{ Total secondary delay } & Effect of \\
& Without rerouting & With rerouting & rerouting \\
\hline FCFS & $774 \min$ & $413 \min$ & $-46.6 \%$ \\
\hline First-order & $688 \min (-11 \%)$ & $436 \min (+6 \%)$ & $-36.6 \%$ \\
Second-order & $357 \min (-54 \%)$ & $259 \min (-37 \%)$ & $-27.5 \%$ \\
\hline DIZ & $375 \min (-52 \%)$ & $252 \min (-39 \%)$ & $-32.8 \%$ \\
\hline
\end{tabular}

Table 4: Comparison of rerouting in conflict prevention techniques based on the total secondary delay.

Table 5 shows the computation time of the different strategies, with and without rerouting. On average, the rerouting optimization includes 1307 variables and 574 constraints. It is clear that the rerouting optimization requires the most computation time within the conflict prevention module. Without rerouting, the computation time of the second-order strategy is five times the computation time of the DIZ heuristic. This very short computation time, especially of the retiming/reordering part of the conflict prevention module, is very important when larger networks or longer heuristic horizons are to be considered. The time spent by the rerouting optimization will remain the same in those cases, but the time spent by the retiming/reordering part will grow rapidly. We expect that this can easily lead to unacceptable computation times for the second-order strategy.

\begin{tabular}{c|cccc}
\hline \multirow{2}{*}{ Strategy } & \multicolumn{4}{|c}{ Computation time } \\
& Without rerouting & With rerouting \\
Average & Maximum & Average & Maximum \\
\hline FCFS & $0.002 \mathrm{~s}$ & $0.2 \mathrm{~s}$ & $0.6 \mathrm{~s}$ & $30.3 \mathrm{~s}$ \\
\hline First-order & $0.4 \mathrm{~s}$ & $3.9 \mathrm{~s}$ & $0.9 \mathrm{~s}$ & $25.5 \mathrm{~s}$ \\
Second-order & $6.4 \mathrm{~s}$ & $8.2 \mathrm{~s}$ & $2.5 \mathrm{~s}$ & $26.8 \mathrm{~s}$ \\
\hline DIZ & $0.4 \mathrm{~s}$ & $3.8 \mathrm{~s}$ & $1 \mathrm{~s}$ & $25.8 \mathrm{~s}$ \\
\hline
\end{tabular}

Table 5: The computation time of the different strategies for $\alpha=60 \%$ with and without rerouting.

\subsubsection{Different delay scenarios}

This section examines the consequences of changing the delay scenarios. Table 6 shows results of 20 random draws from delay scenarios with $\alpha=20 \%, 40 \%$ and 80\%, compared to the standard delay scenario $\alpha=60 \%$. No clear differences are determined between the delay scenarios: the relative improvements compared to FCFS remain similar. The computation times are also similar and therefore are not presented in a table. 


\begin{tabular}{|c|c|c|c|c|}
\hline \multirow[t]{2}{*}{ Strategy } & \multicolumn{4}{|c|}{ Total secondary delay } \\
\hline & $20 \%$ & $40 \%$ & $60 \%$ & $80 \%$ \\
\hline FCFS & $643 \min$ & $799 \min$ & $774 \mathrm{~min}$ & $941 \min$ \\
\hline First-order + rerouting & $337 \min (-48 \%)$ & $473 \min (-41 \%)$ & $436 \min (-44 \%)$ & $518 \min (-45 \%)$ \\
\hline Second-order + rerouting & $230 \min (-64 \%)$ & $238 \min (-70 \%)$ & $259 \min (-67 \%)$ & $269 \min (-71 \%)$ \\
\hline DIZ + rerouting & $217 \min (-66 \%)$ & $246 \min (-69 \%)$ & $252 \min (-67 \%)$ & $277 \min (-71 \%)$ \\
\hline
\end{tabular}

Table 6: Comparison of different $\alpha$ 's for conflict prevention techniques with rerouting based on secondary delay.

\subsubsection{Change in threshold value for offline calculations}

This section examines the effect of changing the threshold value used in the offline calculations. As described in Section 6.3.1, the threshold value determines most likely conflicts. A conflict is most likely if it occurs in at least $50 \%$ of the offline calculations. However, this percentage can be changed to investigate the effect on the performance of the conflict prevention strategy.

Table 7 shows the effect of altering the threshold value to $30 \%$ and $70 \%$ on the total secondary delay. There are in total 98 and 24 most likely conflicts considered in the case of $30 \%$ and $70 \%$ threshold, respectively. Table 8 shows the average computation time of one conflict prevention strategy for the different threshold values. The total secondary delay slightly improves while the computation time slightly increases, when the threshold value decreases. Therefore, $50 \%$ has been agreed as a reasonable trade-off between the quality of the results and the computation time.

\begin{tabular}{c|ccc}
\hline Strategy & \multicolumn{4}{|c}{ Total secondary delay } \\
& $30 \%$ & $50 \%$ & $70 \%$ \\
\hline DIZ & $248 \min (-1.6 \%)$ & $\mathbf{2 5 2} \mathbf{~ m i n}$ & $274 \min (+8.7 \%)$ \\
\hline
\end{tabular}

Table 7: Comparison of altering the threshold value of the most likely conflicts.

\begin{tabular}{c|cccccc}
\hline Strategy & \multicolumn{5}{|c}{ Computation time } \\
& $30 \%$ & $50 \%$ & $70 \%$ & $30 \%$ & $50 \%$ & $70 \%$ \\
\hline DIZ & $1.1 \mathrm{~s}$ & $\mathbf{1} \mathbf{~ s}$ & $0.9 \mathrm{~s}$ & $25.9 \mathrm{~s}$ & $\mathbf{2 5 . 8} \mathbf{s}$ & $25.6 \mathrm{~s}$ \\
\hline
\end{tabular}

Table 8: The computation time of the DIZ heuristic for different threshold values.

\subsection{Performance of the heuristic compared to an exact method}

In order to further analyze the performance of the DIZ heuristic, we compare it to an exact method. The rerouting optimization is not considered in this section because that 
optimization is always solved to optimality and because of long calculation times. Apart from this, we use an exact method considering all possible combinations of resolutions for all conflicts. This allows us to compare the total secondary delay obtained, on the one hand, by resolving each conflict separately in a few seconds by our heuristic, and on the other hand, by calculating afterwards the optimal combination of resolutions for all conflicts together, knowing all train delays and conflicts occurring. Thus, the exact method has perfect information of the complete simulation, which is impossible to implement in practice. Resolving the conflicts sequentially in a closed loop setup works in a more realistic setting where only partial knowledge of the operations is known at any time and where strict time constraints exist about which operations can actually be changed.

To begin with, we tried to find the optimal resolution for each conflict using branchand-bound for the entire network and all trains, for the delay scenario $\alpha=20 \%$ and for a simulation horizon of 30 minutes. Note that this method does not calculate a lower bound. This calculation ran for 216 hours after which it was stopped long before the optimal solution was found. The gap between the best found solution with the exact method and our DIZ heuristic is $11.7 \%$, calculated as $\frac{H E U R-E X}{E X}$, with HEUR the result of the heuristic strategy and EX the result from the exact method.

In order to obtain more reliable results and a proven optimal solution, it was necessary to limit the network and the simulation horizon considered in this comparison. The network is limited to the line Brugge-Gent, including these stations. This limited network is indicated in Figure 12 by the red ellipse. This line is approximately $50 \mathrm{~km}$ long, with 8 smaller stations in between. Clearly, the structure is much less complex. The simulation horizon is limited to 30 minutes, from 7 a.m. to 7.30 a.m., for a total of 84 trains considered. Other parameters and the entire closed loop setup remain unchanged.

The exact method uses a branch-and-bound algorithm for finding the optimal solution. Figure 16 shows the gap for 20 runs, five for each delay scenario, with the line indicating the average value of this strategy. Clearly, the gap between FCFS and exact is the largest and between the second-order strategy and the exact method is the smallest. However, our DIZ heuristic renders good results. On average, the DIZ heuristic has a gap with the exact method of $5.5 \%$ and the maximal gap is $16.4 \%$. The first-order strategy has an average gap of $15.9 \%$ and the second-order strategy $2.8 \%$. 


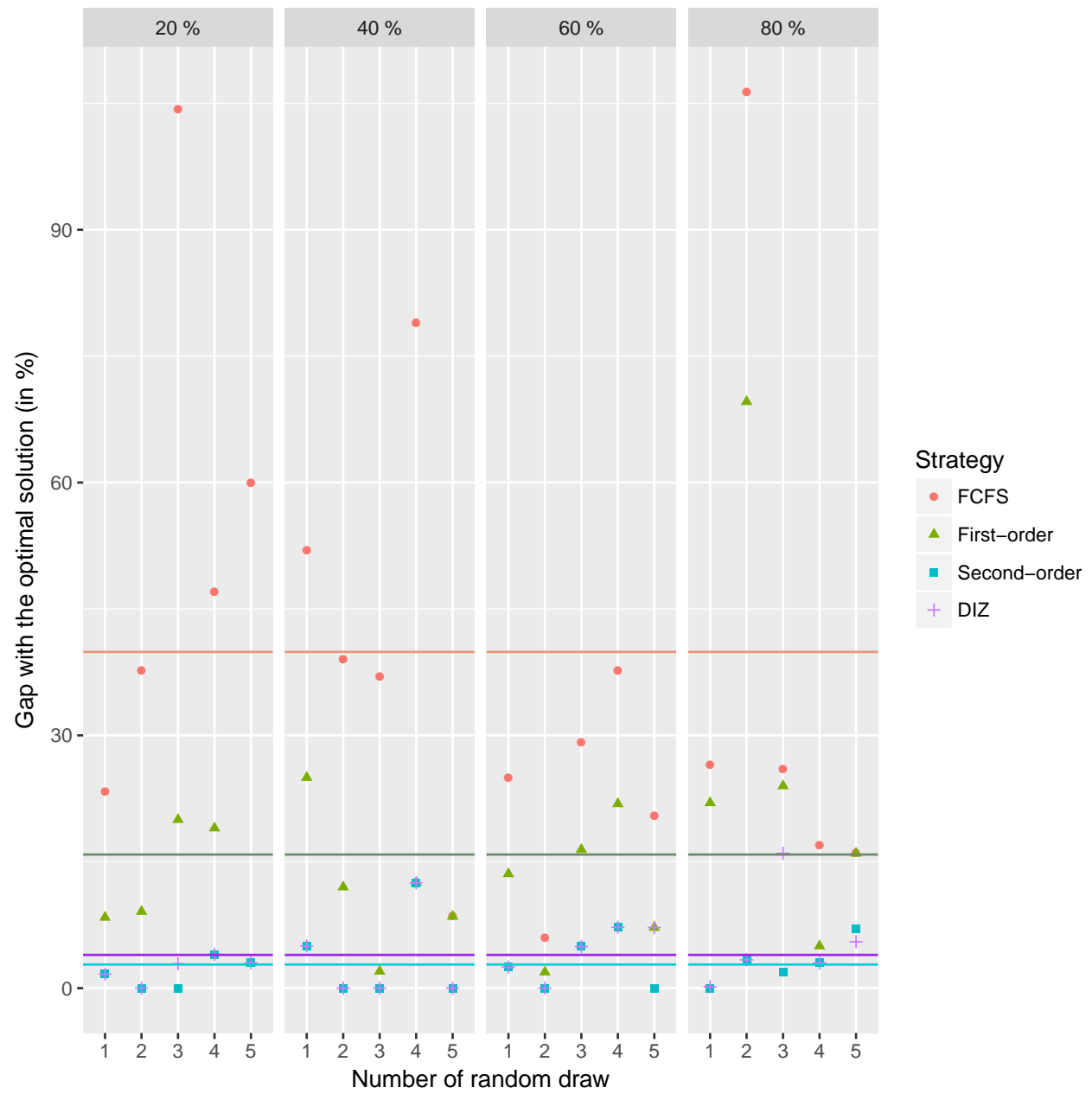

Figure 16: Gap with the exact solution for different delay scenarios and all strategies.

\section{Conclusions and further work}

This paper proposes a conflict prevention strategy that is capable of resolving railway conflicts in real-time for large and complex networks. In those networks, simple heuristic approaches, or a FCFS approach, fail in considering the bigger picture, while complex optimization approaches are stuck with a large computational complexity, hindering optimality of solutions and computation speed. Our Conflict Prevention Strategy (CPS) includes a rerouting optimization that finds optimal alternative routes in station areas, and a Dynamic Impact Zone (DIZ) heuristic, that delays one of the conflicting trains. Both the rerouting optimization and the DIZ heuristic aim to minimize the total secondary delay on all trains in the network.

We evaluate the DIZ heuristic in a realistic setup similar to a Traffic Management System, including a prediction module and a conflict detection module. Whenever a conflict is detected in a station area, the rerouting optimization is started. If rerouting does not 
resolve the conflict or if the conflict takes place in a non-station area, the DIZ heuristic is started. This heuristic relies on dynamic impact zones, based on offline calculations. These offline calculations determine which conflicts are the most likely to occur. When a conflict is subsequently detected in real-time, a dynamic impact zone is built based on the current situation and these most likely conflicts. Then, the progress of all trains within the impact zone is examined during the next 30 minutes in order to determine which retiming/reordering solution results in the least total secondary delay. Both the rerouting optimization and the DIZ heuristic contribute significantly in decreasing the total secondary delay compared to a basic FCFS strategy. Moreover, by comparing with other strategies, we show that the offline calculations and the resulting dynamic impact zone are worthwhile contributions, allowing to scale up the presented conflict prevention strategy to larger networks or to a longer time horizon. Finally, the conflict prevention module directly interacts with a Traffic Management System concerning timetable and microscopic infrastructure data. Moreover, it can also be easily integrated in practice, due to a control delay implemented while optimizing. Therefore, the module and the resulting solutions could be easily implemented in such a system.

Extensive experiments on a large part of the Belgian rail network show that the CPS is capable of decreasing the total secondary delay by more than $67 \%$ compared to a basic dispatching strategy. Also, when comparing our DIZ heuristic with an exact method, on a limited network and with a smaller simulation horizon, results show an average gap of only $5.5 \%$ and a maximum gap of $16.4 \%$. Moreover, the average computation time of the CPS remains low, less than two seconds on average. So, resolving each conflict separately in a few seconds by our heuristic performs only $5.5 \%$ worse than calculating, after knowing all train delays and conflicts occurring, the optimal combination of resolutions for all conflicts together.

As a next step, it should be examined how this dynamic impact zone can be further optimized, for instance by increasing the percentage of the considered conflicts that actually need to be resolved during the progress of the DIZ heuristic. In order to be used for a national network, the size of the impact zone and the type of conflicts considered should be examined. Anyway, the scalability of our approach is an important advantage when considering larger networks. Also, during the progress examination of the current DIZ heuristic, conflicts are resolved using FCFS. This can possibly be further improved by taking into account, for each conflict, the best found resolution from the offline calculations. More complex dynamics can be somehow included in the simulator of operations, like speed adjustments, probability of failures, and/or interaction with passengers flows. Finally, an exact method, able to deal with large and complex networks, would allow to better analyze the performance of our strategy and to improve it further.

\section{Acknowledgements}

We thank the Belgian railway infrastructure manager Infrabel for their commitment during the research and for providing data. 


\section{References}

Borndörfer, R., Klug, T., Lamorgese, L., Mannino, C., Reuther, M., and Schlechte, T. Recent success stories on integrated optimization of railway systems. Transportation Research Part C, 74:196-211, 2017.

Cacchiani, V., Huisman, D., Kidd, M., Kroon, L., Toth, P., Veelenturf, P., and Wagenaar, J. An overview of recovery models and algorithms for real-time railway rescheduling. Transportation Research Part B, 63:15-37, 2014.

Caimi, G., Fuchsberger, M., Laumanns, M., and Lüthi, M. A model predictive control approach for discrete-time rescheduling in complex central railway station areas. Computers \& Operations Research, 39:2578-2593, 2012.

Corman, F. and Meng, L. A review of online dynamic models and algorithms for railway traffic control. IEEE International Conference on Intelligent Rail Transportation (ICIRT), 2013, Beijing, pages 128-133, 2013.

Corman, F. and Quaglietta, E. Closing the loop in railway traffic control: framework design and impacts on operations. Transportation Research Part C, 54:15-39, 2015.

Corman, F., D'Ariano, A., Longo, G., and Medeossi, G. Robustness of advanced train dispatching solutions under disturbances. In Proceedings of WCTR 2010. Lisbon, Portugal, 11-15 July 2010, 2010a.

Corman, F., D'Ariano, A., Pacciarelli, D., and Pranzo, M. A tabu search algorithm for rerouting trains during rail operations. Transportation Research Part B, 44:175-192, 2010b.

Corman, F., D'Ariano, A., Pacciarelli, D., and Pranzo, M. Centralized versus distributed systems to reschedule trains in two dispatching areas. Public Transport, 2(3):219-247, 2010c.

Corman, F., D'Ariano, A., Pacciarelli, D., and Pranzo, M. Optimal inter-area coordination of train rescheduling decisions. Transportation Research Part E, 48:71-88, 2012a.

Corman, F., D'Ariano, A., Pacciarelli, D., and Pranzo, M. Bi-objective conflict detection and resolution in railway traffic management. Transportation Research Part C, 20:79-94, $2012 b$.

Corman, F., D'Ariano, A., Marra, A., Pacciarelli, D., and Samà, M. Integrating train scheduling and delay management in real-time railway traffic control. Transportation Research Part E, 105:213-239, 2017.

D'Ariano, A., Pranzo, M., and Hansen, I. Conflict resolution and train speed coordination for solving real-time timetable perturbations. IEEE Transactions on Intelligent Transportation Systems, 8(2):208-222, 2007. 
D'Ariano, A., Corman, F., Pacciarelli, D., and Pranzo, M. Reordering and Local Rerouting Strategies to Manage Train Traffic in Real Time. Transportation Science, 42(4):405-419, 2008.

Dolder, U., Krista, M., and Voelcker, M. Res - rail control system, realtime train run simulation and conflict detection on a netwide scale based on updated train positions. In Proceedings of the 3rd International Seminar on Railway Operations Modelling and Analysis, RailZurich, 2009.

Hansen, I. and Pachl, J. Railway timetable and traffic: analysis, modelling, simulation. Eurail Press, 2008.

Kecman, P., Corman, F., D'Ariano, A., and Goverde, R. Rescheduling models for railway traffic management in large-scale networks. Public Transport, 5(1):95-123, 2013.

Lamorgese, L. and Mannino, C. An exact decomposition approach for the real-time train dispatching problem. Operations Research, 63(1):48-64, 2015.

Lamorgese, L., Mannino, C., and Piacentini, M. Optimal train dispatching by Benders'-like reformulation. Transportation Science, 50(3):910-925, 2016.

Luan, X., Corman, F., Wang, Y., Meng, L., and Lodewijks, G. Integrated optimization of traffic management and train control for rail networks. In Proceedings of 7th International Seminar on Railway Operations Modelling and Analysis (IAROR), RailLille. Lille, France, 4-7 April 2017, 2017.

Mascis, A. and Pacciarelli, D. Job-shop scheduling with blocking and no-wait constraints. European Journal of Operations Research, 143:498-517, 2002.

Mazzarello, M. and Ottaviani, E. A traffic management system for real-time traffic optimisation in railways. Transportation Research Part B, 41:246-274, 2007.

Meng, L. and Zhou, X. Robust single-track train dispatching model under a dynamic and stochastic environment: A scenario-based rolling horizon solution approach. Transportation Research Part B, 45(7):1080-1102, 2011.

Meng, L. and Zhou, X. Simultaneous train rerouting and rescheduling on a N-track network: a model reformulation with network-based cumulative flow variables. Transportation Research Part B, 67:208-234, 2014.

Pellegrini, P., Marlière, G., and Rodriguez, J. Optimal train routing and scheduling for managing traffic perturbations in complex junctions. Transportation Research Part B, 59:58-80, 2014a.

Pellegrini, P., Marlière, G., and Rodriguez, J. Optimal train routing and scheduling in case of traffic perturbations: Improving solution time through parameter tuning. Transport Research Arena 2014, Paris, 2014b. 
Pellegrini, P., Marlière, G., and Rodriguez, J. A detailed analysis of the actual impact of real-time railway traffic management optimization. Journal of Rail Transport Planning \& Management, 6:13-31, 2016.

Quaglietta, E., Pellegrini, P., Goverde, R., Albrecht, T., Jaekel, B., Marlière, G., Rodriguez, J., Dollevoet, T., Ambrogio, B., Carcasole, D., Giaroli, M., and Nicholson, G. The ON-TIME real-time railway traffic management framework: A proof-of-concept using a scalable standardised data communication architecture. Transportation Research Part C, 63:23-50, 2016.

Samà, M., Pellegrini, P., D’Ariano, A., Rodriguez, J., and Pacciarelli, D. Ant colony optimization for the real-time train routing selection problem. Transportation Research Part B, 85:89-108, 2016.

Samà, M., D'Ariano, A., Corman, F., and Pacciarelli, D. A variable neighbourhood search for fast train scheduling and routing during disturbed railway traffic situations. Computers $\&$ Operations Research, 78:480-499, 2017.

Sels, P., Dewilde, T., Cattrysse, D., and Vansteenwegen, P. Reducing the passenger travel time in practice by the automated construction of a robust railway timetable. Transportation Research Part B, 84:124-156, 2016.

Sobieraj, S., Marlière, G., and Rodriguez, J. Simulation of solutions of a fixed-speed model for the real-time railway traffic optimization problem. In Proceedings of 4 th International Seminar on Railway Operations Modelling and Analysis (IAROR), RailRome. Rome, Italy, 16-18 February 2011, 2011.

Törnquist, J. Railway traffic disturbance management: an experimental analysis of disturbance complexity, management objectives and limitations in planning horizon. Transportation Research Part A, 41(3):249-266, 2007.

Van Thielen, S., Corman, F., and Vansteenwegen, P. An efficient heuristic for train rescheduling and local rerouting. In Proceedings of 7th International Seminar on Railway Operations Modelling and Analysis (IAROR), RailLille. Lille, France, 4-7 April 2017, pages 585-606, 2017.

Wang, P. and Goverde, R. Multi-train trajectory optimization for energy efficiency and delay recovery on single-track railway lines. Transportation Research Part B, 105:340361, 2017.

Wegele, S. and Schnieder, E. Automated dispatching of train operations using genetic algorithms. Computers in Railways IX. WIT Press, Southampton, UK, pages 775-784, 2004. 